\title{
Non-existence of bi-infinite geodesics in the exponential corner growth model
}

\author{
Márton Balázs ${ }^{1}$, Ofer Busani ${ }^{2}$ and Timo Seppäläinen ${ }^{3}$ \\ ${ }^{1}$ University of Bristol, School of Mathematics, Fry Building, Woodland Rd., Bristol BS8 1UG, UK, \\ E-mail: m.balazs@bristol.ac.uk; https://people.maths.bris.ac.uk/ mb13434/. \\ ${ }^{2}$ University of Bristol, School of Mathematics, Fry Building, Woodland Rd., Bristol BS8 1UG, UK, \\ E-mail: o.busani@bristol.ac.uk; https://people.maths.bris.ac.uk/ di18476/. \\ ${ }^{3}$ University of Wisconsin-Madison, Mathematics Department, Van Vleck Hall, 480 Lincoln Dr., Madison, WI \\ 53706-1388, USA, E-mail: seppalai@math.wisc.edu; http://www.math.wisc.edu/ seppalai.
}

Received: 3 April 2020; Revised: 6 May 2020; Accepted: 26 June 2020

2020 Mathematics Subject Classification: Primary - 60K35; Secondary - 60K37

Keywords and phrases: bi-infinite, corner growth model, directed percolation, geodesic, random growth model, last-passage percolation, queues

\begin{abstract}
This paper gives a self-contained proof of the non-existence of nontrivial bi-infinite geodesics in directed planar lastpassage percolation with exponential weights. The techniques used are couplings, coarse graining, and control of geodesics through planarity and estimates derived from increment-stationary versions of the last-passage percolation process.
\end{abstract}

\section{Contents}

1 Introduction $\quad 2$

1.1 Bi-infinite geodesics in random growth . . . . . . . . . . . . . . . 2

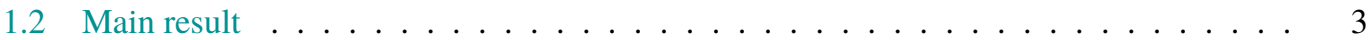

1.3 Related work . . . . . . . . . . . . . . . . . . 4

1.4 Notation and conventions . . . . . . . . . . . . . . . . 4

2 Outline of the proof $\quad 5$

3 Stationary last-passage percolation $\quad 7$

4 Bounds for geodesic fluctuations $\quad 10$

5 No bi-infinite geodesic away from the axes $\quad 16$

6 No nontrivial axis-directed geodesic $\quad 26$

$\begin{array}{lll}\text { Appendix A Queues } & 29\end{array}$

$\begin{array}{lll}\text { Appendix B Coupling and monotonicity in last-passage percolation } & 31\end{array}$

Appendix C Random walk bounds

(C) The Author(s), 2020. Published by Cambridge University Press. This is an Open Access article, distributed under the terms of the Creative Commons Attribution licence (http://creativecommons.org/licenses/by/4.0/), which permits unrestricted re-use, distribution, and reproduction in any medium, provided the original work is properly cited. 


\section{Introduction}

\subsection{Bi-infinite geodesics in random growth}

Since their inception over 50 years ago in the work of Eden [14] and Hammersley and Welsh [20], random growth models have been central drivers of the mathematical theory of spatial random processes. Particularly important classes of growth models are undirected first-passage percolation (FPP) and directed last-passage percolation (LPP), where growth proceeds along optimal paths called geodesics. The structure of these geodesics has been a challenging object of study.

Under natural assumptions, the existence of a geodesic between two points in space is straightforward. A compactness argument gives the existence of a semi-infinite geodesic: that is, a one-sided infinite path that furnishes the geodesic between any two of its points. The existence or non-existence of biinfinite geodesics has turned out to be a very hard problem. This question was first posed to $\mathrm{H}$. Kesten by H. Furstenberg in the context of FPP [25, page 258]. Apart from its significance for random growth, this existence issue is tied to questions about ground states of certain disordered models of statistical physics ([4, page 105], [29, Chapter 1]).

The development of mathematical techniques for infinite geodesics in two-dimensional FPP began with the work of C. Newman and coauthors in the 1990s [28]. Licea and Newman [26] ruled out directed bi-infinite geodesics with given direction in an unknown set of full Lebesgue measure. Much more recently, a bi-infinite geodesic in any fixed direction has been ruled out, but subject to a local regularity condition on the limit shape, by [17] in LPP and by [1, 12] in FPP. The new approach in these works was based on Busemann functions. Bi-infinite FPP geodesics have also been ruled out in certain restricted subsets of the lattice, such as half-planes [3,36]. However, despite all the effort, a feasible strategy for solving the bi-infinite existence problem in FPP without restrictive assumptions is not presently visible.

In the seminal paper of Johansson [23], the Tracy-Widom distribution of the limit fluctuation in LPP was proved for geometric and exponential weights. This led to a large literature on exactly solvable models in the Kardar-Parisi-Zhang (KPZ) class and gave rise to a new subject, integrable probability. Deep results on exactly solvable models have identified the limiting objects, the KPZ fixed point [27], and the directed landscape [13], and provide a benchmark for the expected behavior of LPP with general weight distributions in accordance with the KPZ universality conjecture. While progress in exactly solvable models in the past 20 years has been striking, for general LPP, basic questions such as regularity of the limit shape and the order of fluctuations remain open. Moreover, the methods of integrable probability are often so specialized that it seems unlikely that they can provide a roadmap for approaching general growth models.

Parallel to the development of integrable probability, a suite of more robust probabilistic techniques for deriving fluctuation bounds evolved. After the seminal paper of Cator and Groeneboom [9] on the Poissonian planar growth model, [5] derived the 1/3 shape exponent and 2/3 transversal exponent in the exponential LPP. These papers point the way to a proof of the KPZ exponents under a strictly concave shape function and sufficiently mixing Busemann functions. This approach has also been successful for a class of zero-range processes that goes beyond exactly solvable models [6].

Ergodic Busemann functions were developed for general LPP in [18]. The point of view was that of queueing theory, where Busemann functions can be identified with fixed points of stationary queues in tandem. The follow-up work [17] proved results about competition interfaces, directed semi-infinite geodesics, and the nonexistence of bi-infinite geodesics in a particular direction, under regularity assumptions on the shape function.

In exactly solvable planar directed LPP, techniques have now improved to the point where the existence problem of bi-infinite geodesics can be given a complete solution. The first proof of nonexistence in planar LPP with exponential weights appeared in the 2018 preprint [7] of Basu, Hoffman, and Sly. Their work relies on fluctuation and moderate deviation estimates for the passage times that come from integrable probability. These estimates were originally obtained through combinatorial analysis, asymptotic analysis of Fredholm determinants, and random matrix methods. Further results from these estimates were derived in the preprint [8] by Basu, Sidoravicius, and Sly, in particular 


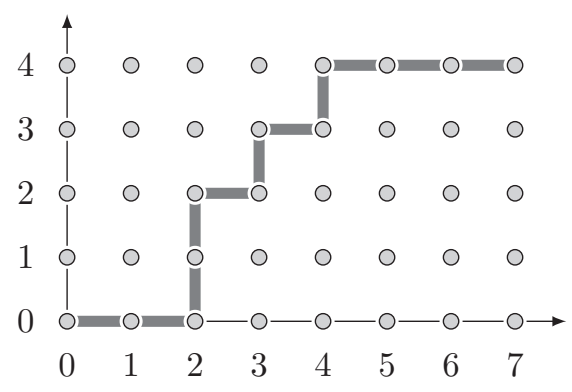

Figure 1.1. An up-right path from $(0,0)$ to $(7,4)$ on the lattice $\mathbb{Z}^{2}$.

to control transversal fluctuations of geodesics, and then applied to the bi-infinite geodesic problem in [7].

The concentration bounds used in [7] should hold for LPP models outside the exactly solvable ones; but it is unclear how these bounds can be obtained from the conjectural basic properties of the general LPP, such as strict concavity of the shape function and strong mixing of the Busemann functions. Our paper does not have a result for general LPP, but our proof does lay out a possible route from fairly basic properties of LPP to the non-existence of bi-infinite geodesics. We do this through deeper insight into the queueing picture obtained in [15]. Going through the proofs in this paper, the reader should see that our choice of the exponential distribution can be viewed as an assumption on the basic properties of the model, conjectured to hold for any continuous distribution with sufficiently decaying moment. Along the way, nothing beyond standard probability tools such as coupling and coarse graining is needed.

Next we state the main result and then relate our proof to existing literature. In particular, we contrast our work with [7] in more detail.

\subsection{Main result}

The model studied is a version of nearest-neighbor directed LPP on the planar integer lattice, also known as the corner growth model (CGM). Let $\omega=\left\{\omega_{x}\right\}_{x \in \mathbb{Z}^{2}}$ be an assignment of random weights on the vertices of $\mathbb{Z}^{2}$. The weights $\omega_{x}$ are independent and identically distributed (i.i.d) random variables with rate one exponential distribution: that is, $\mathbb{P}\left(\omega_{x}>t\right)=e^{-t}$ for each $x \in \mathbb{Z}^{2}$ and real $t \geq 0$. The last-passage value $G_{x, y}$ for coordinatewise ordered points $x \leq y$ on $\mathbb{Z}^{2}$ is defined by

$$
G_{x, y}=\max _{x_{\bullet} \in \Pi_{x, y}} \sum_{k=0}^{|y-x|_{1}} \omega_{x_{k}},
$$

where $\Pi_{x, y}$ is the set of nearest-neighbor up-right paths $x_{\bullet}=\left(x_{k}\right)_{k=0}^{n}$ that start at $x_{0}=x$ and end at $x_{n}=y$, with $n=|y-x|_{1}=$ the number of nearest-neighbor steps from $x$ to $y$. Such paths are defined by the requirement $x_{k+1}-x_{k} \in\left\{e_{1}, e_{2}\right\}$. (See Figure 1.1.) When the weights have a continuous distribution such as the exponential, (1.1) has a unique maximizing path $\pi^{x, y} \in \Pi_{x, y}$ called the ( point-to-point or finite) geodesic.

A bi-infinite geodesic is a nearest-neighbor up-right path $\left\{x_{k}\right\}_{k \in \mathbb{Z}}$ indexed by all integers, with the property that for all $m<n$, the path segment $x_{[m, n]}=\left\{x_{k}\right\}_{k=m}^{n}$ is the geodesic between $x_{m}$ and $x_{n}$. A straight line $\left\{x_{k}=x+k e_{i}\right\}_{k \in \mathbb{Z}}$, for $x \in \mathbb{Z}^{2}$ and $i \in\{1,2\}$, is trivially a bi-infinite geodesic because there are no alternative paths between any two of its points. Let us call a bi-infinite geodesic nontrivial if it is not of this type. The main result is that the exponential CGM has no nontrivial bi-infinite geodesics.

Theorem 1.1. Assume that weights have i.i.d. exponential distribution. Then with probability one, there are no nontrivial bi-infinite geodesics. 


\subsection{Related work}

Among past work on geodesics, our proof is in spirit aligned with the Damron-Hanson work on FPP $[11,12]$ and with the general LPP work in $[17,22]$, in the sense that the stationary version of the process lies at the heart of the matter. Compared to earlier work on the exponential CGM that utilized couplings with the stationary version, such as [5,31,35], two specific new developments made this paper possible:

(i) The discovery in [15] of the stationary distribution of the joint LPP process with multiple characteristic directions. A bivariate version of this distribution is constructed in Theorem 3.1 below.

(ii) A novel argument for controlling the location of the geodesic by coupling the bulk process with two distinct stationary processes from two different directions (Lemma 5.5 below).

One can be fairly confident that these features extend to both zero-temperature and positivetemperature polymer models in $1+1$ dimensions that possess a tractable stationary version. This includes various last-passage models in both discrete and continuous space, such as those studied in $[2,19,24,30,33,34]$, and the four currently known solvable polymer models [10]. In positivetemperature polymer models, the analogous question concerns the existence of bi-infinite Gibbs measures, as discussed in [21]. These matters are left for future work.

As in [7] by Basu, Hoffman, and Sly, our proof comes in two parts:

(a) The main argument rules out bi-infinite geodesics with finite positive slope.

(b) An easier argument shows that no geodesic can come infinitely often arbitrarily close to an axis in the macroscopic scale.

Beyond this superficial similarity, the two proofs are quite different in both parts (a) and (b).

Our part (a) in Section 5 is a straightforward estimation of the probability that a geodesic through the origin connects the boundaries of a square at scale $N$. By contrast, [7] controls complicated events that involve coalescence of geodesics. This yields additional results of interest, but the simplicity of the bi-infinite geodesic problem is obscured. Their sharper tools give a better estimate of the probability of a connection through the origin, namely $O\left(N^{-1 / 3}\right)$, while our cruder bound is $O\left(N^{-1 / 24}\right)$. In Remark 5.6 we indicate the precise place where our estimates grow beyond optimal order of magnitude.

Part (b) in [7] utilizes fluctuations. Our part (b) in Section 6 uses the limit shape and planarity.

We conclude this introduction by observing that the non-existence of bi-infinite geodesics will be a tool for further results. To cite an example, article [22] studies a random graph in the CGM that represents an analogue of shocks in Hamilton-Jacobi equations. Theorem 4.3 in [22] shows that the absence of bi-infinite geodesics implies certain coalescence properties of this 'instability graph'.

Section 2 outlines the proof of Theorem 1.1 and describes the organization of the rest of the paper. We provide a self-contained exposition of the entire proof, including proof sketches of many auxiliary results that we use. We collect below some notation for easy reference.

\subsection{Notation and conventions}

$\mathbb{Z}_{\geq 0}=\{0,1,2,3, \ldots\}$ and $\mathbb{Z}_{>0}=\{1,2,3, \ldots\}$. For real numbers $a$ and $b, a \vee b=\max \{a, b\}$ and $\llbracket a, b \rrbracket=[a, b] \cap \mathbb{Z}$. 0 denotes the origin of both $\mathbb{R}$ and $\mathbb{R}^{2} . C(\varepsilon)$ and $N_{0}(\varepsilon)$ are constants that depend on a parameter $\varepsilon$, but their values can change from line to line.

For $x=\left(x_{1}, x_{2}\right), y=\left(y_{1}, y_{2}\right) \in \mathbb{R}^{2}$, we use the following conventions. The standard basis vectors are $e_{1}=(1,0)$ and $e_{2}=(0,1)$. The $\ell^{1}$-norm is $|x|_{1}=\left|x_{1}\right|+\left|x_{2}\right|$. Integer parts and inequalities are interpreted coordinatewise: $\lfloor x\rfloor=\left(\left\lfloor x_{1}\right\rfloor,\left\lfloor x_{2}\right\rfloor\right)$, and $x \leq y$ means $x_{1} \leq y_{1}$ and $x_{2} \leq y_{2}$. Notation $[x, y]$ represents both the line segment $[x, y]=\{t x+(1-t) y: 0 \leq t \leq 1\}$ and the rectangle $[x, y]=\left\{\left(z_{1}, z_{2}\right) \in \mathbb{R}^{2}: x_{i} \leq z_{i} \leq y_{i}\right.$ for $\left.i=1,2\right\}$. The context makes clear which one is used. An open line segment is $] x, y[=\{t x+(1-t) y: 0<t<1\}$. The lattice rectangle and line segment are denoted by $\llbracket x, y \rrbracket=[x, y] \cap \mathbb{Z}^{2}$. Path segments are abbreviated by $\pi_{[m, n]}=\left(\pi_{i}\right)_{i=m}^{n}$. 


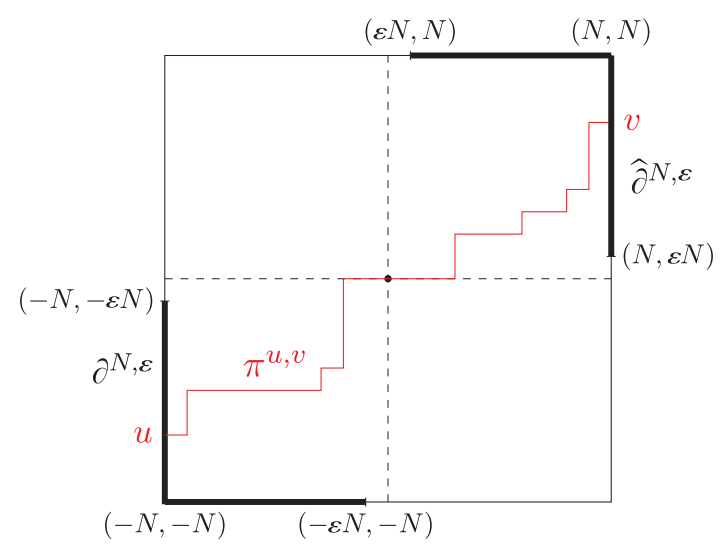

Figure 2.1. The event $W_{N}, \varepsilon$. The thickset portions of the boundary are $\partial^{N, \varepsilon}$ and $\widehat{\partial}^{N, \varepsilon}$. They are connected by the geodesic $\pi^{u, v}$ through the origin.

$\bar{X}=X-E X$ denotes a random variable $X$ centered at its mean. $X \sim \operatorname{Exp}(\lambda)$ for $0<\lambda<\infty$ means the random variable $X$ has exponential distribution with rate $\lambda$ : in other words, $P(X>t)=e^{-\lambda t}$ for $t \geq 0$.

\section{Outline of the proof}

We state two auxiliary theorems and use them to prove Theorem 1.1. Then we sketch the main ideas behind the auxiliary theorems and explain the organization of the rest of the paper.

By the shift-invariance of the underlying weight distribution, it suffices to prove that with probability one, no nontrivial bi-infinite geodesic goes through the origin. This task is split into two cases: either the geodesic ultimately stays away from the axes on a macroscopic scale, or it comes infinitely often macroscopically close to some axis.

For the first case, for large positive integers $N$ and small $\varepsilon>0$, we rule out geodesics that connect the southwest boundary of the lattice square $\llbracket-N, N \rrbracket^{2}$ to its northeast boundary through the origin and whose empirical average slope is in the range $\left[\varepsilon, \varepsilon^{-1}\right]$. Define these portions of the boundary of the square: in the southwest

$$
\partial^{N, \varepsilon}=(\{-N\} \times \llbracket-N,-\varepsilon N \rrbracket) \cup(\llbracket-N,-\varepsilon N \rrbracket \times\{-N\})
$$

and in the northeast

$$
\widehat{\partial}^{N, \varepsilon}=(\{N\} \times \llbracket \varepsilon N, N \rrbracket) \cup(\llbracket \varepsilon N, N \rrbracket \times\{N\}) .
$$

Define the following event, illustrated in Figure 2.1:

$$
\begin{aligned}
& W_{N, \varepsilon}=\left\{\exists \text { points } u \in \partial^{N, \varepsilon} \text { and } v \in \widehat{\partial}^{N, \varepsilon}\right. \text { such that } \\
& \text { the geodesic } \left.\pi^{u, v} \text { goes through the origin }\right\} .
\end{aligned}
$$

We have the following quantitative control of this event.

Theorem 2.1. For each $\varepsilon>0$, there exists a constant $C(\varepsilon)>0$ such that $\mathbb{P}\left(W_{N, \varepsilon}\right) \leq C(\varepsilon) N^{-\frac{1}{24}}$ for all $N \geq 1$.

Theorem 2.1 rules out all geodesics that stay macroscopically away from the axes. The next theorem shows that there are no nontrivial geodesics that come macroscopically arbitrarily close to an axis. 
Theorem 2.2. The following statement holds with probability one. For $i \in\{1,2\}$ and each $x \in \mathbb{Z}_{\geq 0}^{2}$, $\left\{x_{k}=x+k e_{i}\right\}_{k \in \mathbb{Z}_{\geq 0}}$ is the only semi-infinite geodesic that satisfies $x_{0}=x$ and $\underline{\lim } k^{-1} x_{k} \cdot e_{3-i}=0$.

We combine the two theorems above to rule out all nontrivial bi-infinite geodesics.

Proof of Theorem 1.1, assuming Theorems 2.1 and 2.2. Fix a positive sequence $\varepsilon_{j} \searrow 0$. Define the event

$$
A=\bigcap_{j \geq 1} \bigcap_{M \geq 1} \bigcup_{N \geq M} W_{N, \varepsilon_{j}}^{c} .
$$

Theorem 2.1 implies that $\mathbb{P}(A)=1$ :

$$
\begin{gathered}
\mathbb{P}\left(A^{c}\right)=\mathbb{P}\left(\bigcup_{j} \bigcup_{M} \bigcap_{N \geq M} W_{N, \varepsilon_{j}}\right) \leq \sum_{j} \mathbb{P}\left(\bigcup_{M} \bigcap_{N \geq M} W_{N, \varepsilon_{j}}\right)=\sum_{j} \lim _{M \rightarrow \infty} \mathbb{P}\left(\bigcap_{N \geq M} W_{N, \varepsilon_{j}}\right) \\
\leq \sum_{j} \lim _{M \rightarrow \infty} \mathbb{P}\left(W_{M, \varepsilon_{j}}\right) \leq \sum_{j} \lim _{M \rightarrow \infty} C\left(\varepsilon_{j}\right) M^{-\frac{1}{24}}=0 .
\end{gathered}
$$

For $i \in\{1,2\}$, let $B_{i}$ be the event that there are no semi-infinite geodesics $\left\{x_{k}\right\}_{k \geq 0}$ such that $x_{0}=0$ and $\underline{\lim }_{k \rightarrow \infty} k^{-1} x_{k} \cdot e_{i}=0$ except for the trivial one, $\left\{x_{k}=k e_{3-i}\right\}_{k \in \mathbb{Z}_{\geq 0}}$. Let $R$ reflect the weight configuration across the origin: $(R \omega)_{x}=\omega_{-x}$ for $x \in \mathbb{Z}^{2}$. Define the event

$$
B=B_{1} \cap B_{2} \cap R^{-1} B_{1} \cap R^{-1} B_{2} .
$$

On the event $B$, every semi-infinite geodesic that either starts or ends at the origin satisfies the condition that, far enough from the origin, it lies entirely inside a closed cone with apex at the origin and disjoint from the coordinate axes. Theorem 2.2 and the reflection invariance of the distribution of the weights $\omega$ imply that $\mathbb{P}(B)=1$.

We claim that on the full-probability event $A \cap B$, there are no nontrivial bi-infinite geodesics through the origin. To show this, suppose there exists a nontrivial bi-infinite geodesic $\pi$ through the origin in the weight configuration $\omega$. Consider the following dichotomy:

(i) $\exists j, M \in \mathbb{Z}_{>0}$ such that $\pi$ connects $\partial^{N, \varepsilon_{j}}$ to $\widehat{\partial}^{N, \varepsilon_{j}}$ for all $N \geq M$, or

(ii) $\forall j, M \in \mathbb{Z}_{>0}, \exists N \geq M$ such that $\pi$ misses either $\partial^{N, \varepsilon_{j}}$ or $\widehat{\partial}^{N, \varepsilon_{j}}$.

Alternative (i) forces $\omega \in A^{c}$. In alternative (ii), if $\pi$ misses $\widehat{\partial}^{N}, \varepsilon_{j}$ infinitely often for each $\varepsilon_{j}$, it follows that $\underline{\lim }_{k \rightarrow \infty} k^{-1} \pi_{k} \cdot e_{i}=0$ for either $i=1$ or 2 . Thus $\omega \in B_{1}^{c} \cup B_{2}^{c}$. Similarly, missing $\partial^{N, \varepsilon_{j}}$ infinitely often for each $\varepsilon_{j}$ implies $R \omega \in B_{1}^{c} \cup B_{2}^{c}$.

Thus a nontrivial bi-infinite geodesic through the origin is possible only on the zero-probability event $A^{c} \cup B^{c}$.

Sketch of the proof of Theorem 2.1. Theorem 2.1 comes from two distinct stages.

(i) In the first stage, the southwest boundary $\partial^{N, \varepsilon}$ is divided into blocks of size $N^{2 / 3}$ and the northeast boundary $\widehat{\partial}^{N, \varepsilon}$ into blocks of size $N^{19 / 24}$. The probability that a geodesic connects two diagonally opposite blocks through the origin is bounded by $N^{-2 / 5}$ (Lemma 5.5). The control here comes from random walk bounds on the location where a geodesic crosses the $y$-axis. These bounds are developed through a coupling with increment-stationary LPP processes.

(ii) The second stage shows that any geodesic that connects an $N^{2 / 3}$-block through the origin to a point outside its opposite $N^{19 / 24}$-block violates the $N^{2 / 3} \mathrm{KPZ}$ wandering exponent. Through another coupling argument, the probability of this happening is bounded by $N^{-3 / 8}$ (Lemma 5.7).

Multiplying by the number of $N^{2 / 3}$-blocks gives the estimate $O\left(N^{1 / 3} \cdot N^{-2 / 5}+N^{1 / 3} \cdot N^{-3 / 8}\right)=$ $O\left(N^{-1 / 24}\right)$. 

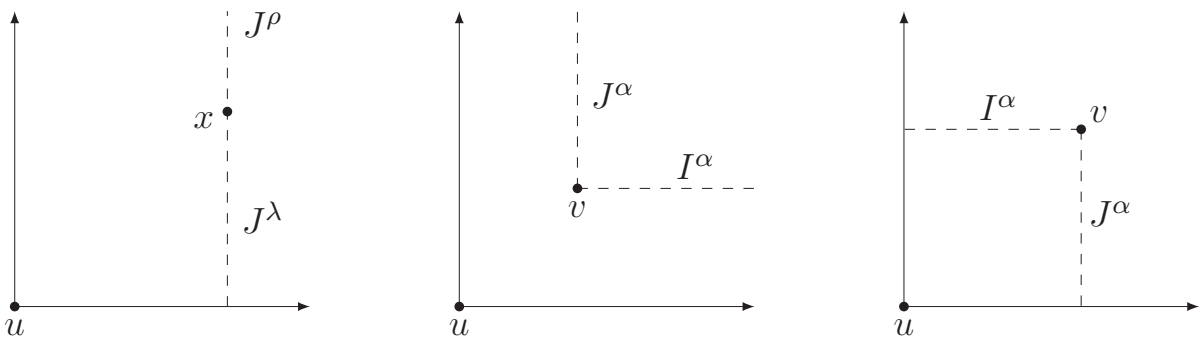

Figure 3.1. The independent increment variables from Theorem 3.1. Left: $J^{\lambda}$ below $x$ and $J^{\rho}$ above $x$ from part (i). Middle and right: $I^{\alpha}$ and $J^{\alpha}$ increments on down-right lattice paths from part (ii).

Sketch of the proof of Theorem 2.2. Comparison with increment-stationary LPP processes shows that the quantity $G_{0, \pi_{n}}-G_{e_{2}, \pi_{n}}$ blows up if $\pi_{n}$ is a path above the $x$-axis but $n^{-1} \pi_{n}$ comes arbitrarily close to the $x$-axis. This rules out the possibility that $\pi$. is a geodesic.

The next two sections develop tools: Section 3 a coupling of increment-stationary LPP processes and Section 4 bounds on geodesic fluctuations. The proof of Theorem 2.1 follows in Section 5 and that of Theorem 2.2 in Section 6.

\section{Stationary last-passage percolation}

Pick $0<\lambda<\rho<1$ and a base vertex $u \in \mathbb{Z}^{2}$. We construct two coupled LPP processes $G_{u, \bullet}^{\lambda}$ and $G_{u, \bullet}^{\rho}$ on the nonnegative quadrant $u+\mathbb{Z}_{\geq 0}^{2}$ such that their increments are jointly stationary under lattice translations. Both processes use the same i.i.d. $\operatorname{Exp}(1)$ weights $\left\{\omega_{x}\right\}_{x \in u+\mathbb{Z}_{>0}^{2}}$ in the bulk. They have boundary conditions on the positive $x$ - and $y$-axes centered at $u$, coupled in a way described in the next theorem.

For $\alpha \in\{\lambda, \rho\}$, the definition of the process $G_{u, \bullet}^{\alpha}$ goes as follows. The boundary weights are denoted by $\left\{I_{u+i e_{1}}^{\alpha}, J_{u+j e_{2}}^{\alpha}: i, j \in \mathbb{Z}_{>0}\right\}$. Put $G_{u, u}^{\alpha}=0$, and on the boundaries

$$
G_{u, u+k e_{1}}^{\alpha}=\sum_{i=1}^{k} I_{i e_{1}}^{\alpha} \quad \text { and } \quad G_{u, u+l e_{2}}^{\alpha}=\sum_{j=1}^{l} J_{j e_{2}}^{\alpha} \quad \text { for } k, l \geq 1 .
$$

In the bulk for $x=\left(x_{1}, x_{2}\right) \in u+\mathbb{Z}_{>0}^{2}$,

$$
\begin{aligned}
G_{u, x}^{\alpha} & =\max _{1 \leq k \leq x_{1}-u_{1}}\left\{\sum_{i=1}^{k} I_{u+i e_{1}}^{\alpha}+G_{u+k e_{1}+e_{2}, x}\right\} \bigvee \max _{1 \leq \ell \leq x_{2}-u_{2}}\left\{\sum_{j=1}^{\ell} J_{u+j e_{2}}^{\alpha}+G_{u+e_{1}+\ell e_{2}, x}\right\} \\
& =G_{u, x-e_{1}}^{\alpha} \vee G_{u, x-e_{2}}^{\alpha}+\omega_{x} .
\end{aligned}
$$

$G_{u, \bullet}^{\alpha}$ does not use a weight at the base point $u$. Inside the braces above, $G_{x, y}$ is the LPP process (1.1) that uses the bulk weights $\omega$. Define increment variables for vertices $x \in u+\mathbb{Z}_{>0}^{2}$ by

$$
I_{x}^{\alpha}=G_{x}^{\alpha}-G_{x-e_{1}}^{\alpha} \quad \text { and } \quad J_{x}^{\alpha}=G_{x}^{\alpha}-G_{x-e_{2}}^{\alpha} .
$$

An important part of the next theorem for the sequel is the independence of various collections of increment variables. These are illustrated in Figure 3.1.

Theorem 3.1. Let $0<\lambda<\rho<1$ and $u \in \mathbb{Z}^{2}$. There exists a coupling of the boundary weights $\left\{I_{u+i e_{1}}^{\lambda}, I_{u+i e_{1}}^{\rho}, J_{u+j e_{2}}^{\lambda}, J_{u+j e_{2}}^{\rho}: i, j \in \mathbb{Z}_{>0}\right\}$ such that the joint process $\left(G_{u, \bullet}^{\lambda}, G_{u, \bullet}^{\rho}\right)$ has the following properties: 
(i) (Joint) The joint process of increments is stationary: for each $v \in u+\mathbb{Z}_{\geq 0}^{2}$,

$$
\left\{\left(G_{u, v+x}^{\lambda}-G_{u, v}^{\lambda}, G_{u, v+x}^{\rho}-G_{u, v}^{\rho}\right): x \in \mathbb{Z}_{\geq 0}^{2}\right\} \stackrel{d}{=}\left\{\left(G_{u, u+x}^{\lambda}, G_{u, u+x}^{\rho}\right): x \in \mathbb{Z}_{\geq 0}^{2}\right\} .
$$

The following independence property holds along vertical lines: for each $x \in u+\mathbb{Z}_{>0}^{2}$, the variables $\left\{J_{x+j e_{2}}^{\lambda}: u_{2}-x_{2}+1 \leq j \leq 0\right\}$ and $\left\{J_{x+j e_{2}}^{\rho}: j \geq 1\right\}$ are mutually independent.

(ii) (Marginal) For both $\alpha \in\{\lambda, \rho\}$ and each $v \in u+\mathbb{Z}_{\geq 0}^{2}$, the increment variables $\left\{I_{v+i e_{1}}^{\alpha}, J_{v+j e_{2}}^{\alpha}\right.$ : $\left.i, j \in \mathbb{Z}_{>0}\right\}$ are mutually independent with marginal distributions

$$
I_{v+i e_{1}}^{\alpha} \sim \operatorname{Exp}(1-\alpha) \quad \text { and } \quad J_{v+j e_{2}}^{\alpha} \sim \operatorname{Exp}(\alpha) .
$$

The same is true of the variables $\left\{I_{v-i e_{1}}^{\alpha}, J_{v-j e_{2}}^{\alpha}: 0 \leq i<v_{1}-u_{1}, 0 \leq j<v_{2}-u_{2}\right\}$.

(iii) (Monotonicity) The boundary weights can be coupled with i.i.d. $\operatorname{Exp}(1)$ weights $\left\{\eta_{u+i e_{1}}, \eta_{u+j e_{2}}\right.$ : $i, j \geq 1\}$ independent of the bulk weights $\omega$ so that these inequalities hold almost surely for all $i, j \geq 1$ :

$$
\eta_{u+i e_{1}} \leq I_{u+i e_{1}}^{\lambda} \leq I_{u+i e_{1}}^{\rho} \text { and } \quad \eta_{u+j e_{2}} \leq J_{u+j e_{2}}^{\rho} \leq J_{u+j e_{2}}^{\lambda}
$$

Proof. We construct a joint LPP process $\left(L_{x}^{\lambda}, L_{x}^{\rho}\right)_{x \in u+\mathbb{Z}_{\geq 0} \times \mathbb{Z}}$ on the discrete right half-plane with origin at $u$. In the interior, we have i.i.d. $\operatorname{Exp}(1)$ weights $\left\{\omega_{x}: x_{1}>u_{1}\right\}$ as before. For $\alpha \in\{\lambda, \rho\}$, let $\mathbf{Y}^{\lambda}=\left\{Y_{j}^{\lambda}\right\}_{j \in \mathbb{Z}}$ and $\mathbf{Y}^{\rho}=\left\{Y_{j}^{\rho}\right\}_{j \in \mathbb{Z}}$ be independent sequences of i.i.d. variables with marginal distributions $Y_{j}^{\alpha} \sim \operatorname{Exp}(\alpha)$, independent of $\omega$. From these, we define the boundary weights $\mathbf{J}^{\lambda}=\left\{J_{u+j e_{2}}^{\lambda}\right\}_{j \in \mathbb{Z}}$ and $\mathbf{J}^{\rho}=\left\{J_{u+j e_{2}}^{\rho}\right\}_{j \in \mathbb{Z}}$ on the $y$-axis through $u$ by the equation $\left(\mathbf{J}^{\rho}, \mathbf{J}^{\lambda}\right)=\left(\mathbf{Y}^{\rho}, D\left(\mathbf{Y}^{\lambda}, \mathbf{Y}^{\rho}\right)\right) . D$ is the departure process operator from (A.1) in Appendix A. This gives a pair of coupled sequences $\left(\mathbf{J}^{\rho}, \mathbf{J}^{\lambda}\right)$. Marginally, $\left\{J_{u+j e_{2}}^{\alpha}\right\}_{j \in \mathbb{Z}}$ are i.i.d. $\operatorname{Exp}(\alpha)$.

For $\alpha \in\{\lambda, \rho\}$, define the LPP values on the $y$-axis by

$$
L_{u}^{\alpha}=0, \quad L_{u+j e_{2}}^{\alpha}-L_{u+(j-1) e_{2}}^{\alpha}=J_{u+j e_{2}}^{\alpha} \quad \text { for } j \in \mathbb{Z} .
$$

This results in negative values $L_{u+j e_{2}}^{\alpha}$ for $j<0$. Complete the definitions by putting, again for $\alpha \in\{\lambda, \rho\}$ and now for $x \in u+\mathbb{Z}_{>0} \times \mathbb{Z}$,

$$
L_{x}^{\alpha}=\sup _{j: j \leq x_{2}-u_{2}}\left\{L_{u+j e_{2}}^{\alpha}+G_{u+e_{1}+j e_{2}, x}\right\}, \quad I_{x}^{\alpha}=L_{x}^{\alpha}-L_{x-e_{1}}^{\alpha} \quad \text { and } \quad J_{x}^{\alpha}=L_{x}^{\alpha}-L_{x-e_{2}}^{\alpha}
$$

The supremum is achieved at a finite $j$ because the boundary variables $J^{\alpha}$ are stochastically larger than the bulk weights. This follows from the distributional properties established in the next paragraph.

For $k \geq 0$, denote the sequences of $J$-increments on the vertical line shifted by $k e_{1}$ from the $y$-axis by

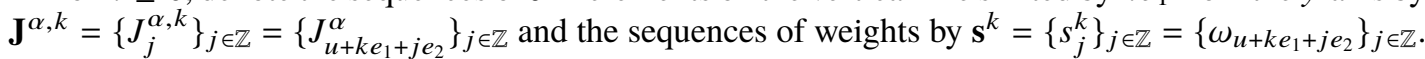
$\mathbf{J}^{\alpha, 0}$ is the original boundary sequence $\mathbf{J}^{\alpha}$ we began with. Then, in terms of Lemma A.2, we have the following. With $\left(\sigma, \alpha_{1}, \alpha_{2}\right)=(1, \rho, \lambda),\left(\mathbf{J}^{\rho}, \mathbf{J}^{\lambda}\right)$ has the distribution of $\left(\mathbf{a}^{1}, \mathbf{a}^{2}\right)$; and for each $k \geq 1$ and $\alpha \in\{\lambda, \rho\}, \mathbf{J}^{\alpha, k}=D\left(\mathbf{J}^{\alpha, k-1}, \mathbf{s}^{k}\right)$. Repeated application of Lemma A.2(iv) implies the distributional equality $\left(\mathbf{J}^{\rho, k}, \mathbf{J}^{\lambda, k}\right) \stackrel{d}{=}\left(\mathbf{J}^{\rho}, \mathbf{J}^{\lambda}\right)$ for all $k \geq 0$. Lemma A.2(v) gives the property that, for any $x \in u+\mathbb{Z}_{\geq 0} \times \mathbb{Z}$, the increment variables

$$
\left\{J_{x+j e_{2}}^{\lambda}: j \leq 0\right\} \quad \text { and } \quad\left\{J_{x+j e_{2}}^{\rho}: j \geq 1\right\} \quad \text { are mutually independent. }
$$

The evolution in (3.6) satisfies a semigroup property: for each $k$, the values $L_{x}^{\alpha}$ for $x_{1} \geq u_{1}+k+1$ satisfy

$$
L_{x}^{\alpha}=\sup _{j: j \leq x_{2}-u_{2}}\left\{L_{u+k e_{1}+j e_{2}}^{\alpha}+G_{u+(k+1) e_{1}+j e_{2}, x}\right\} .
$$


It follows that the entire process of increments is invariant under translations that keep it in the halfspace: for $z \in \mathbb{Z}_{\geq 0} \times \mathbb{Z}$,

$$
\begin{array}{r}
\left\{I_{z+x+e_{1}}^{\lambda}, I_{z+x+e_{1}}^{\rho}, J_{z+x}^{\lambda}, J_{z+x}^{\rho}: x \in u+\mathbb{Z}_{\geq 0} \times \mathbb{Z}\right\} \\
\stackrel{d}{=}\left\{I_{x+e_{1}}^{\lambda}, I_{x+e_{1}}^{\rho}, J_{x}^{\lambda}, J_{x}^{\rho}: x \in u+\mathbb{Z}_{\geq 0} \times \mathbb{Z}\right\} .
\end{array}
$$

(The index is $x+e_{1}$ rather than $x$ in the $I$-increments simply because these are not defined on the boundary where $x_{1}=u_{1}$.)

We claim that for $\alpha \in\{\lambda, \rho\}$ and for any new base point $v \in u+\mathbb{Z}_{\geq 0} \times \mathbb{Z}$,

$$
\begin{gathered}
\left\{I_{v+i e_{1}}^{\alpha}, J_{v+j e_{2}}^{\alpha}: i, j \in \mathbb{Z}_{>0}\right\} \text { are mutually independent with marginal distributions } \\
I_{v+i e_{1}}^{\alpha} \sim \operatorname{Exp}(1-\alpha) \text { and } J_{v+j e_{2}}^{\alpha} \sim \operatorname{Exp}(\alpha) .
\end{gathered}
$$

Since everything is shift-invariant, we can take $v=u$. As observed above, $\mathbf{J}^{\alpha}$ is a sequence of i.i.d. $\operatorname{Exp}(\alpha)$ random variables by Lemma A.2(i). Thus it suffices to prove the marginal statement about $\left\{I_{u+i e_{1}}^{\alpha}: i \geq 1\right\}$ because these variables are a function of $\left\{J_{u+j e_{2}}^{\alpha}, \omega_{u+(i, j)}: i \geq 1, j \leq 0\right\}$, which are independent of $\left\{J_{u+j e_{2}}^{\alpha}: j \geq 1\right\}$.

The claim for $\left\{I_{u+i e_{1}}^{\alpha}: i \geq 1\right\}$ follows from proving inductively the following statement for each $n \geq 1$ :

$$
\begin{aligned}
& \left\{I_{u+i e_{1}}^{\alpha}, J_{u+n e_{1}+j e_{2}}^{\alpha}: 1 \leq i \leq n, j \leq 0\right\} \text { are mutually independent with } \\
& \text { marginal distributions } I_{u+i e_{1}}^{\alpha} \sim \operatorname{Exp}(1-\alpha) \text { and } J_{u+n e_{1}+j e_{2}}^{\alpha} \sim \operatorname{Exp}(\alpha) .
\end{aligned}
$$

Begin with the case $n=1$. From the inputs given by inter-arrival times $\left\{a_{j}=J_{u+j e_{2}}^{\alpha}: j \leq 0\right\}$ and service times $\left\{s_{j}=\omega_{u+e_{1}+j e_{2}}: j \leq 0\right\}$, equations (A.3) compute the inter-departure times $\left\{d_{j}=J_{u+e_{1}+j e_{2}}^{\alpha}: j \leq 0\right\}$ and the sojourn time $t_{0}=I_{u+e_{1}}^{\alpha}$. Part of Lemma A.2(ii) then gives exactly statement (3.10) for $n=1$. (The dual-service variables $\check{s}_{j}$ that also appear in Lemma A.2(ii) are not needed here.)

Continue inductively. Assume that (3.10) holds for a given $n$. Then feed to the queueing operators inter-arrival times $\left\{a_{j}=J_{u+n e_{1}+j e_{2}}^{\alpha}: j \leq 0\right\}$ and service times $\left\{s_{j}=\omega_{u+(n+1) e_{1}+j e_{2}}: j \leq 0\right\}$, all independent of $\left\{I_{u+i e_{1}}^{\alpha}: 1 \leq i \leq n\right\}$. Compute the inter-departure times $\left\{d_{j}=J_{u+(n+1) e_{1}+j e_{2}}^{\alpha}: j \leq 0\right\}$ and the sojourn time $t_{0}=I_{u+(n+1) e_{1}}^{\alpha}$. Lemma A.2(ii) extends the validity of (3.10) to $n+1$. Claim (3.9) has been verified.

To prove Theorem 3.1, take the coupled boundary weights $\left\{I_{u+i e_{1}}^{\alpha}, J_{u+j e_{2}}^{\alpha}: i, j \geq 1, \alpha \in\{\lambda, \rho\}\right\}$ as constructed above. The LPP process $\left\{G_{u, x}^{\alpha}: x \in u+\mathbb{Z}_{\geq 0}^{2}\right\}$ defined by (3.1)-(3.2) is then exactly the same as the restriction $\left\{L_{x}^{\alpha}: x \in u+\mathbb{Z}_{\geq 0}^{2}\right\}$ of $L^{\alpha}$. Namely, (3.2) can be rewritten as follows:

$$
\begin{aligned}
G_{u, x}^{\alpha}= & \max _{1 \leq k \leq x_{1}-u_{1}}\left\{L_{u+k e_{1}}^{\alpha}+G_{u+k e_{1}+e_{2}, x}\right\} \bigvee \max _{1 \leq \ell \leq x_{2}-u_{2}}\left\{L_{u+\ell e_{2}}^{\alpha}+G_{u+e_{1}+\ell e_{2}, x}\right\} \\
= & \sup _{j \leq 0}\left\{L_{u+j e_{2}}^{\alpha}+\max _{1 \leq k \leq x_{1}-u_{1}}\left[G_{u+e_{1}+j e_{2}, u+k e_{1}}+G_{u+k e_{1}+e_{2}, x}\right]\right\} \\
& \bigvee_{1 \leq \ell \leq x_{2}-u_{2}}\left\{L_{u+\ell e_{2}}^{\alpha}+G_{u+e_{1}+\ell e_{2}, x}\right\} \\
= & \operatorname{mup}_{j: j \leq x_{2}-u_{2}}\left\{L_{u+j e_{2}}^{\alpha}+G_{u+e_{1}+j e_{2}, x}\right\}=L_{x}^{\alpha} .
\end{aligned}
$$

Invariance (3.4) comes from (3.8). The statement in part (i) about independence comes from (3.7). The first statement of part (ii) of the theorem comes from (3.9) and the second statement from (3.10).

As the last step, we prove part (iii). The inequality $J_{u+j e_{2}}^{\rho} \leq J_{u+j e_{2}}^{\lambda}$ comes directly from (A.4), due to the construction $\left(\mathbf{J}^{\rho}, \mathbf{J}^{\lambda}\right)=\left(\mathbf{Y}^{\rho}, D\left(\mathbf{Y}^{\lambda}, \mathbf{Y}^{\rho}\right)\right)$. Then (A.5) gives the inequality $I_{u+i e_{1}}^{\lambda} \leq I_{u+i e_{1}}^{\rho}$ because, in terms of the notation used above, the sequence $\mathbf{I}^{\alpha, k}=\left\{I_{u+k e_{1}+j e_{2}}^{\alpha}\right\}_{j \in \mathbb{Z}}$ satisfies $\mathbf{I}^{\alpha, k}=S\left(\mathbf{J}^{\alpha, k-1}, \mathbf{s}^{k}\right)$. 
Define

$$
\eta_{u+i e_{1}}=(1-\lambda) I_{u+i e_{1}}^{\lambda} \text { and } \quad \eta_{u+j e_{2}}=\rho J_{u+j e_{2}}^{\rho} \text { for } \quad i, j \geq 1 .
$$

Inequalities (3.5) hold now. By the already proved part (ii) of the theorem, marginally $\left\{\eta_{u+i e_{1}}\right\}_{i \geq 1}$ are i.i.d. $\operatorname{Exp}(1)$ and $\left\{\eta_{u+j e_{2}}\right\}_{j \geq 1}$ are also i.i.d. $\operatorname{Exp}(1)$. These two sequences are independent of each other because the weights $\left\{\eta_{u+i e_{1}}\right\}_{i \geq 1}=\left\{(1-\lambda) I_{u+i e_{1}}^{\lambda}\right\}_{i \geq 1}$ are functions of $\left\{J_{u+j e_{2}}^{\lambda}, \omega_{u+(i, j)}: i \geq 1, j \leq 0\right\}$, and these latter are independent of $\left\{J_{u+j e_{2}}^{\rho}: j \geq 1\right\}$, by construction and by (3.7). Part (iii) is proved.

\section{Bounds for geodesic fluctuations}

Let $G_{u, \bullet}^{\rho}$, be a stationary LPP process with base point $u$ as described in Theorem 3.1, with independent boundary weights $I_{u+i e_{1}} \sim \operatorname{Exp}(1-\rho)$ and $J_{u+j e_{2}} \sim \operatorname{Exp}(\rho)$ for $i, j \geq 1$. For a northeast endpoint $x \in u+\mathbb{Z}_{>0}^{2}$, let $Z_{u, x}^{\rho}$ be the signed exit point of the geodesic $\pi_{\bullet}^{\rho, u, x}$ of $G_{u, x}^{\rho}$ from the west and south boundaries of $u+\mathbb{Z}_{\geq 0}^{2}$. More precisely,

$$
Z_{u, x}^{\rho}= \begin{cases}\underset{k}{\arg \max }\left\{\sum_{i=1}^{k} I_{u+i e_{1}}+G_{u+k e_{1}+e_{2}, x}\right\}, & \text { if } \pi_{1}^{\rho, u, x}=u+e_{1}, \\ \underset{\ell}{\arg \max }\left\{\sum_{j=1}^{\ell} J_{u+j e_{2}}+G_{u+\ell e_{2}+e_{1}, x}\right\}, & \text { if } \pi_{1}^{\rho, u, x}=u+e_{2} .\end{cases}
$$

The open line segment of interior directions is denoted by $] e_{2}, e_{1}[=\{(s, 1-s): 0<s<1\}$. The parameter $\rho \in(0,1)$ of the stationary LPP process is in one-to-one correspondence with a direction vector $\left.\xi=\left(\xi_{1}, 1-\xi_{1}\right) \in\right] e_{2}, e_{1}[$ through these equations:

$$
\xi=\xi(\rho)=\left(\frac{(1-\rho)^{2}}{(1-\rho)^{2}+\rho^{2}}, \frac{\rho^{2}}{(1-\rho)^{2}+\rho^{2}}\right) \Longleftrightarrow \rho=\rho(\xi)=\frac{\sqrt{1-\xi_{1}}}{\sqrt{\xi_{1}}+\sqrt{1-\xi_{1}}} .
$$

Direction $\xi(\rho)$ is called the characteristic direction associated with the parameter $\rho$. A key property that distinguishes $\xi(\rho)$ among all $\eta \in] e_{2}, e_{1}\left[\right.$ is that $\left|Z_{u, u+\lfloor N \eta\rfloor}^{\rho}\right|=o(N)$ almost surely if and only if $\eta=\xi(\rho)$. Write the characteristic direction as

$$
\xi(\rho)=\left(\xi_{1}(\rho), \xi_{2}(\rho)\right)=\alpha[\rho]\left((1-\rho)^{2}, \rho^{2}\right)
$$

by introducing

$$
\alpha[\rho]=\frac{1}{(1-\rho)^{2}+\rho^{2}} .
$$

Note the bounds $1 \leq \alpha[\rho] \leq 2$.

This section derives basic estimates for later use. We take the base point as the origin $u=0$, but in later applications the base point will vary. Abbreviate the sum of boundary weights on the $x$-axis as $S_{k}^{\rho}=\sum_{i=1}^{k} I_{i e_{1}}^{\rho}=G_{0, k e_{1}}^{\rho}$. The starting point for the estimates is the variance formula of the next theorem.

Theorem 4.1. For $(m, n) \in \mathbb{Z}_{>0}^{2}$,

$$
\operatorname{Var}\left[G_{0,(m, n)}^{\rho}\right]=-\frac{m}{(1-\rho)^{2}}+\frac{n}{\rho^{2}}+\frac{2}{1-\rho} \mathbb{E}\left[S_{\left(Z_{0,(m, n)}^{\rho}\right)^{+}}^{\rho}\right] .
$$

Sketch of proof. We give the main steps of the argument. Detailed proofs appear in Lemma 4.6 of [5] and in Section 5.3 of [35]. Utilizing

$$
G_{0,(m, n)}^{\rho}=\sum_{i=1}^{m} I_{(i, 0)}^{\rho}+\sum_{j=1}^{n} J_{(m, j)}^{\rho}=\sum_{j=1}^{n} J_{(0, j)}^{\rho}+\sum_{i=1}^{m} I_{(i, n)}^{\rho}
$$


and the independence of $\left\{I_{(i, n)}^{\rho}, J_{(m, j)}^{\rho}: 1 \leq i \leq m, 1 \leq j \leq n\right\}$ from Theorem 3.1(ii), deduce

$$
\operatorname{Var}\left[G_{0,(m, n)}^{\rho}\right]=-\operatorname{Var}\left[\sum_{i=1}^{m} I_{(i, n)}^{\rho}\right]+\operatorname{Var}\left[\sum_{j=1}^{n} J_{(0, j)}^{\rho}\right]+2 \operatorname{Cov}\left[\sum_{i=1}^{m} I_{(i, 0)}^{\rho}, \sum_{i=1}^{m} I_{(i, n)}^{\rho}\right]
$$

The first two terms of (4.4) and (4.5) match. Let $I_{x}^{\lambda, \rho}$ be increment variables (3.3) for a process whose independent boundary weights satisfy $I_{(i, 0)}^{\lambda, \rho} \sim \operatorname{Exp}(\lambda)$ and $J_{(0, j)}^{\lambda, \rho} \sim \operatorname{Exp}(\rho)$. Complete the proof through

$$
\operatorname{Cov}\left[\sum_{i=1}^{m} I_{(i, 0)}^{\rho}, \sum_{i=1}^{m} I_{(i, n)}^{\rho}\right]=-\left.\frac{\partial}{\partial \lambda} \mathbb{E}\left[\sum_{i=1}^{m} I_{(i, n)}^{\lambda, \rho}\right]\right|_{\lambda=1-\rho}=\frac{1}{1-\rho} \mathbb{E}\left[S_{\left(Z_{0,(m, n)}^{\rho}\right)^{+}}^{\rho}\right] .
$$

The line above comes by calculating the middle derivative in two ways. For the left equality, condition on $\sum_{i=1}^{m} I_{(i, 0)}^{\lambda, \rho}$ and differentiate its density. For the right equality, express the boundary variables $I_{(i, 0)}^{\lambda, \rho}$ as functions of uniform random variables and take the differentiation inside the expectation.

Next we derive a bound on the exit point. This CGM result is from [5], which adapted the seminal result from [9]. A proof also appears in Section 5.4 of [35].

Theorem 4.2. For $0<\varepsilon<\frac{1}{2}$ and $\kappa>0$, there exists a finite constant $B(\varepsilon, \kappa)$ such that

$$
\mathbb{P}\left\{\left|Z_{0,(m, n)}^{\rho}\right| \geq \ell\right\} \leq B(\varepsilon, \kappa)\left(\frac{N^{2}}{\ell^{3}}+\frac{N^{8 / 3}}{\ell^{4}}\right) \text { for all } m, n, N, \ell \geq 1
$$

whenever $\rho \in[\varepsilon, 1-\varepsilon]$ and $|(m, n)-N \xi(\rho)|_{1} \leq \kappa$.

Proof. It suffices to prove the bound

$$
\mathbb{P}\left\{Z_{0,(m, n)}^{\rho} \geq \ell\right\} \leq B(\varepsilon, \kappa)\left(\frac{N^{2}}{\ell^{3}}+\frac{N^{8 / 3}}{\ell^{4}}\right)
$$

because the other probability $\mathbb{P}\left\{Z_{0,(m, n)}^{\rho} \leq-\ell\right\}$ is obtained by reflection across the diagonal. We can assume that $\ell \leq m$, for otherwise the probability in (4.7) vanishes. Let $0<r<1$ be a constant that will be set small enough in the proof. Let

$$
\lambda=\rho+\frac{r \ell}{N}
$$

We take $r=r(\varepsilon, \kappa)$ at least small enough that $r m / N<\frac{1}{2}(1-\rho)$ for $m \leq N(1-\rho)^{2}+\kappa$ and $N \geq 1$. This guarantees that $\lambda \in\left(\rho, \frac{1+\rho}{2}\right)$ is also a legitimate parameter for an increment-stationary CGM.

Couple the boundary weights so that $I_{i e_{1}}^{\lambda} \geq I_{i e_{1}}^{\rho}$. In the first inequality below, use $S_{k}^{\lambda}+G_{(k, 1),(m, n)} \leq$ $G_{0,(m, n)}^{\lambda}$. The second equality follows from $I_{i e_{1}}^{\lambda} \geq I_{i e_{1}}^{\rho}$. Recall that $\bar{X}=X-\mathbb{E} X$.

$$
\begin{aligned}
\mathbb{P} & \left\{Z_{0,(m, n)}^{\rho} \geq \ell\right\}=\mathbb{P}\left\{\exists k \geq \ell: S_{k}^{\rho}+G_{(k, 1),(m, n)}=G_{0,(m, n)}^{\rho}\right\} \\
& \leq \mathbb{P}\left\{\exists k \geq \ell: S_{k}^{\lambda}-S_{k}^{\rho} \leq G_{0,(m, n)}^{\lambda}-G_{0,(m, n)}^{\rho}\right\} \\
& =\mathbb{P}\left\{S_{\ell}^{\lambda}-S_{\ell}^{\rho} \leq G_{0,(m, n)}^{\lambda}-G_{0,(m, n)}^{\rho}\right\} \\
& =\mathbb{P}\left\{\overline{S_{\ell}^{\lambda}}-\overline{S_{\ell}^{\rho}} \leq \overline{G_{0,(m, n)}^{\lambda}}-\overline{G_{0,(m, n)}^{\rho}}-\left(\mathbb{E}\left[S_{\ell}^{\lambda}-S_{\ell}^{\rho}\right]-\mathbb{E}\left[G_{0,(m, n)}^{\lambda}-G_{0,(m, n)}^{\rho}\right]\right)\right\} .
\end{aligned}
$$

Compute and bound the means in the last probability above.

$$
\mathbb{E}\left[S_{\ell}^{\lambda}-S_{\ell}^{\rho}\right]=\ell\left(\frac{1}{1-\lambda}-\frac{1}{1-\rho}\right)=\frac{\ell}{(1-\lambda)(1-\rho)}(\lambda-\rho)=\frac{1}{(1-\lambda)(1-\rho)} \cdot \frac{r \ell^{2}}{N}
$$


Introduce the quantities $\kappa_{N}^{1}=m-N \xi_{1}(\rho)$ and $\kappa_{N}^{2}=n-N \xi_{2}(\rho)$ that satisfy $\left|\kappa_{N}^{1}\right|+\left|\kappa_{N}^{2}\right| \leq \kappa$. Then for the means of the LPP values,

$$
\begin{aligned}
\mathbb{E} & {\left[G_{0,(m, n)}^{\lambda}-G_{0,(m, n)}^{\rho}\right]=m\left(\frac{1}{1-\lambda}-\frac{1}{1-\rho}\right)+n\left(\frac{1}{\lambda}-\frac{1}{\rho}\right) } \\
& =\left(\frac{m}{(1-\lambda)(1-\rho)}-\frac{n}{\lambda \rho}\right)(\lambda-\rho) \\
& =\alpha[\rho] N\left(\frac{1-\rho}{1-\lambda}-\frac{\rho}{\lambda}\right)(\lambda-\rho)+\left(\frac{\kappa_{N}^{1}}{(1-\lambda)(1-\rho)}-\frac{\kappa_{N}^{2}}{\lambda \rho}\right)(\lambda-\rho) \\
& =\frac{\alpha[\rho] N}{\lambda(1-\lambda)}(\lambda-\rho)^{2}+\left(\frac{\kappa_{N}^{1}}{(1-\lambda)(1-\rho)}-\frac{\kappa_{N}^{2}}{\lambda \rho}\right)(\lambda-\rho) \\
& =\frac{\alpha[\rho] r^{2} \ell^{2}}{\lambda(1-\lambda) N}+\left(\frac{\kappa_{N}^{1}}{(1-\lambda)(1-\rho)}-\frac{\kappa_{N}^{2}}{\lambda \rho}\right) \frac{r \ell}{N} \\
& \leq \frac{\alpha[\rho] r^{2} \ell^{2}}{\lambda(1-\lambda) N}+C_{1}(\varepsilon, \kappa) \frac{r \ell}{N}
\end{aligned}
$$

Comparison of (4.9) and (4.10) shows that if we choose $r$ and $c_{3}$ small enough as functions of $(\varepsilon, \kappa)$, then there is a constant $\ell_{0}(\varepsilon, \kappa) \geq 1$ such that for $\ell \geq \ell_{0}(\varepsilon, \kappa)$ and $\rho \in[\varepsilon, 1-\varepsilon]$, we have

$$
\mathbb{E}\left[S_{\ell}^{\lambda}-S_{\ell}^{\rho}\right]>\mathbb{E}\left[G_{0,(m, n)}^{\lambda}-G_{0,(m, n)}^{\rho}\right]+c_{3} \frac{r \ell^{2}}{N} .
$$

We continue the bound on $\mathbb{P}\left\{Z_{0,(m, n)}^{\rho} \geq \ell\right\}$ from line (4.8) and apply (4.11). Below, we pack the $(\varepsilon, \kappa)$-dependent factors into a constant $C=C(\varepsilon, \kappa)$.

$$
\begin{aligned}
\mathbb{P}\{ & \left.Z_{0,(m, n)}^{\rho} \geq \ell\right\} \leq \mathbb{P}\left\{\overline{S_{\ell}^{\lambda}}-\overline{S_{\ell}^{\rho}} \leq \overline{G_{0,(m, n)}^{\lambda}}-\overline{G_{0,(m, n)}^{\rho}}-c_{3} \frac{r \ell^{2}}{N}\right\} \\
& \leq \mathbb{P}\left\{\overline{S_{\ell}^{\lambda}}-\overline{S_{\ell}^{\rho}} \leq-c_{3} \frac{r \ell^{2}}{2 N}\right\}+\mathbb{P}\left\{\overline{G_{0,(m, n)}^{\lambda}}-\overline{G_{0,(m, n)}^{\rho}} \geq c_{3} \frac{r \ell^{2}}{2 N}\right\} \\
& \leq \frac{C N^{2}}{\ell^{4}} \operatorname{Var}\left[S_{\ell}^{\lambda}-S_{\ell}^{\rho}\right]+\frac{C N^{2}}{\ell^{4}} \operatorname{Var}\left[G_{0,(m, n)}^{\lambda}-G_{0,(m, n)}^{\rho}\right] \\
& \leq \frac{C N^{2}}{\ell^{3}}+\frac{C N^{2}}{\ell^{4}}\left(\mathbb{V a r}\left[G_{0,(m, n)}^{\lambda}\right]+\mathbb{V a r}\left[G_{0,(m, n)}^{\rho}\right]\right) \\
& \leq \frac{C N^{2}}{\ell^{3}}+\frac{C N^{2}}{\ell^{4}}\left(\mathbb{V a r}\left[G_{0,(m, n)}^{\rho}\right]+m(\lambda-\rho)\right) \\
& =\frac{C N^{2}}{\ell^{3}}+\frac{C N^{2}}{\ell^{4}}\left(-\frac{m}{(1-\rho)^{2}}+\frac{n}{\rho^{2}}+\frac{2}{1-\rho} \mathbb{E}\left[S_{Z_{0,(m, n)}^{\rho}}^{\rho}\right]+\left((1-\rho)^{2} N+\kappa\right) \cdot \frac{r \ell}{N}\right) \\
& \leq \frac{C N^{2}}{\ell^{3}}+\frac{C N^{2}}{\ell^{4}}\left(\mathbb{E}\left[Z_{0,(m, n)}^{\rho}\right]+\ell\right) \leq \frac{C N^{2}}{\ell^{3}}+\frac{C N^{2}}{\ell^{4}} \mathbb{E}\left[Z_{0,(m, n)}^{\rho}\right] .
\end{aligned}
$$

Along the way, we used the following two inequalities. For $\varepsilon \leq \rho \leq \lambda \leq 1-\varepsilon / 2$,

$$
\operatorname{Var}\left[G_{0,(m, n)}^{\lambda}\right] \leq \operatorname{Var}\left[G_{0,(m, n)}^{\rho}\right]+C m(\lambda-\rho)
$$

holds by the variance formula (4.4) [35, Lemma 5.7]. Next, even though the i.i.d. terms $I_{i e_{1}}^{\rho}$ are positively correlated with $Z_{0,(m, n)}^{\rho}$, we have the bound

$$
\mathbb{E}\left[S_{\left(Z_{0,(m, n)}^{\rho}\right)^{+}}^{\rho}\right] \leq C\left(\mathbb{E}\left[\left(Z_{0,(m, n)}^{\rho}\right)^{+}\right]+1\right)
$$

because the terms $I_{i e_{1}}^{\rho}$ have high moments [35, Lemma 5.8]. 
Define a constant $b=\ell_{0}+C$, with $\ell_{0}(\varepsilon, \kappa)$ determined above (4.11), and $C(\varepsilon, \kappa)$ from line (4.12) above. Then

$$
\begin{aligned}
\mathbb{E}\left[Z_{0,(m, n)}^{\rho}\right] & =\int_{0}^{m} \mathbb{P}\left(Z_{0,(m, n)}^{\rho} \geq s\right) d s \leq b N^{2 / 3}+C \int_{b N^{2 / 3}}^{\infty}\left(\frac{N^{2}}{s^{3}}+\frac{N^{2}}{s^{4}} \mathbb{E}\left[Z_{0,(m, n)}^{\rho}\right]\right) d s \\
& =b N^{2 / 3}+\frac{C N^{2 / 3}}{2 b^{2}}+\frac{C}{3 b^{3}} \mathbb{E}\left[Z_{0,(m, n)}^{\rho}\right] \leq b N^{2 / 3}+\frac{1}{2} N^{2 / 3}+\frac{1}{3} \mathbb{E}\left[Z_{0,(m, n)}^{\rho}\right] .
\end{aligned}
$$

From this, we obtain the bound $\mathbb{E}\left[Z_{0,(m, n)}^{\rho}\right] \leq C_{1}(\varepsilon, \kappa) N^{2 / 3}$. Substituting this back into line (4.12) gives the conclusion (4.7) for $\ell \geq \ell_{0}(\varepsilon, \kappa)$. By increasing the constant $B(\varepsilon, \kappa)$, we can cover all $\ell \geq 1$.

We state a corollary that quantifies the effect of deviating the endpoint from the characteristic direction.

Corollary 4.3. For $0<\varepsilon<\frac{1}{2}$ and $\kappa>0$, there exists a finite constant $C(\varepsilon, \kappa)$ such that for $m, n, N$, $b \geq 1$,

$$
\mathbb{P}\left\{Z_{0,\left(m, n+\left\lfloor b N^{2 / 3}\right\rfloor\right)}^{\rho} \geq 1\right\} \leq C(\varepsilon, \kappa) b^{-3}
$$

and

$$
\mathbb{P}\left\{Z_{0,\left(m, n-\left\lfloor b N^{2 / 3}\right\rfloor\right)}^{\rho} \leq-1\right\} \leq C(\varepsilon, \kappa) b^{-3}
$$

whenever these conditions hold: $\rho \in[\varepsilon, 1-\varepsilon],|(m, n)-N \xi(\rho)|_{1} \leq \kappa$, and, in the case of (4.14), also $n-\left\lfloor b N^{2 / 3}\right\rfloor \geq 1$.

Proof. For (4.13), introduce another scaling parameter $M$ and a constant $d$ via

$$
M \xi_{2}(\rho)=n+b N^{2 / 3} \text { and } \quad d=b\left(\frac{1-\rho}{\rho}\right)^{2} \geq b \varepsilon^{2} .
$$

Then $\left\lfloor M \xi_{2}(\rho)\right\rfloor=n+\left\lfloor b N^{2 / 3}\right\rfloor$ while

$$
M \xi_{1}(\rho)=\frac{n(1-\rho)^{2}}{\rho^{2}}+d N^{2 / 3}=m+d N^{2 / 3}+\frac{n(1-\rho)^{2}-m \rho^{2}}{\rho^{2}},
$$

from which follows

$$
\left\lfloor M \xi_{1}(\rho)\right\rfloor \geq m+\left\lfloor b \varepsilon^{2} N^{2 / 3}\right\rfloor-\kappa \varepsilon^{-2} .
$$

By the shifting Lemma B.4 in Appendix B,

$$
\begin{gathered}
\mathbb{P}\left\{Z_{0,\left(m, n+\left\lfloor b N^{2 / 3}\right\rfloor\right)}^{\rho} \geq 1\right\} \leq \mathbb{P}\left\{Z_{0,\left(\left\lfloor M \xi_{1}(\rho)\right\rfloor,\left\lfloor M \xi_{2}(\rho)\right\rfloor\right)}^{\rho} \geq b \varepsilon^{2} N^{2 / 3}-\kappa \varepsilon^{-2}\right\} \\
\leq \mathbb{P}\left\{Z_{0,\left(\left\lfloor M \xi_{1}(\rho)\right\rfloor,\left\lfloor M \xi_{2}(\rho)\right\rfloor\right)}^{\rho} \geq \frac{1}{2} b \varepsilon^{2} N^{2 / 3}\right\} \leq C(\varepsilon) b^{-3} .
\end{gathered}
$$

In the second-last inequality we assumed $b \geq 2 \kappa \varepsilon^{-4}$ which entails no loss of generality because we can adjust $C(\varepsilon, \kappa)$. The last inequality is from the upper bound (4.6).

For bound (4.14) apply again Lemma B.4 in Appendix B and then the upper bound (4.6):

$$
\mathbb{P}\left\{Z_{0,\left(m, n-\left\lfloor b N^{2 / 3}\right\rfloor\right)}^{\rho} \leq-1\right\} \leq \mathbb{P}\left\{Z_{0,(m, n)}^{\rho} \leq-b N^{2 / 3}\right\} \leq C(\varepsilon, \kappa) b^{-3} .
$$

For directions $\left.\xi=\left(\xi_{1}, \xi_{2}\right) \in\right] e_{2}, e_{1}[, x$-coordinates $m \in \mathbb{Z}$, and $r>0$, define

$$
\mathcal{C}_{m, r}^{\xi}=\{m\} \times\left\{y \in \mathbb{Z}:\left|m \xi_{2} / \xi_{1}-y\right| \leq r N^{\frac{2}{3}}\right\} .
$$




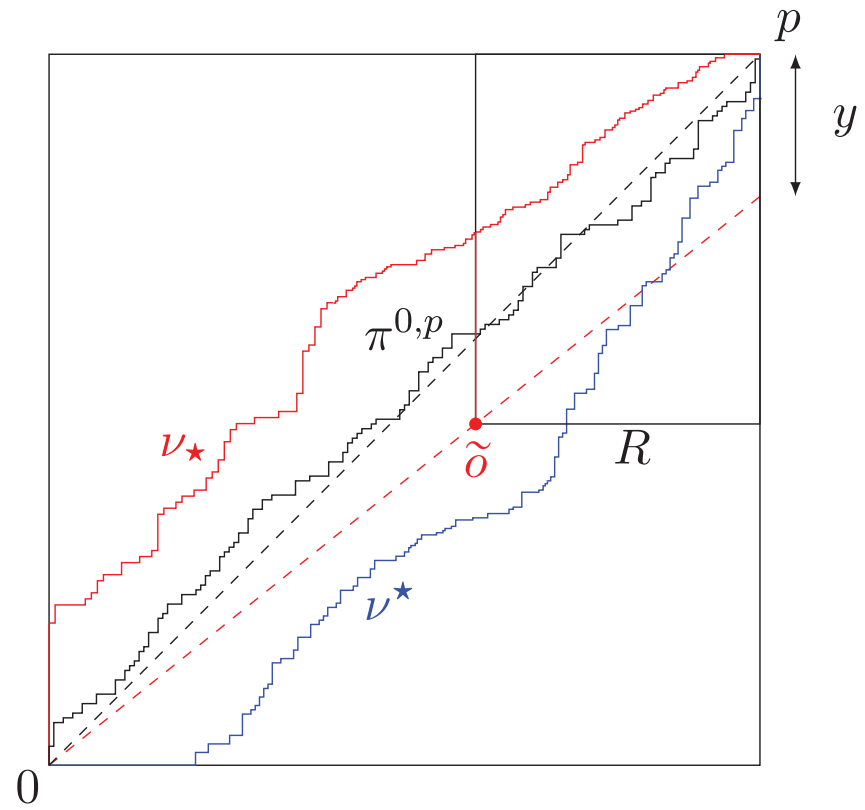

Figure 4.1. Illustration of the proof of Lemma 4.4. On the event $\left\{Z_{0, p}^{\rho_{\star}}<0, Z_{0, p}^{\rho^{\star}}>0\right\}$, geodesic $v_{\star}$ exits off the $y$-axis and $v^{\star}$ off the $x$-axis. Dashed straight lines: $[0, p]$ is the ray in direction $\xi$, [0, $\left.\widetilde{o}\right]$ in direction $\xi_{\star}$. With high probability, the geodesics $v_{\star}$ and $v^{\star}$ sandwich the geodesic $\pi^{0, p}$, while not wandering too far from the $\xi$-directed ray.

$\mathcal{C}_{m, r}^{\xi}$ is the vertical line segment of length $2 r N^{2 / 3}$ centered on the $\xi$-directed ray at point $\left(m, m \xi_{2} / \xi_{1}\right)$. Recall that $\pi^{0, p}$ denotes the unique geodesic of $G_{0, p}$ that uses i.i.d. $\operatorname{Exp}(1)$ weights. The next lemma shows that for large $r$, the geodesic $\pi^{0,\lfloor\xi N\rfloor}$ is very likely to intersect $\mathcal{C}_{m, r}^{\xi}$.

Lemma 4.4. For $0<\delta, \varepsilon<\frac{1}{3}$, there exists a finite constant $C=C(\delta, \varepsilon)$ such that the following holds for all $N \geq 1$ and $1 \leq r<\frac{\sqrt{\varepsilon}}{2(1+\sqrt{\varepsilon})} N^{1 / 3}$ : for any direction $\left.\xi=\left(\xi_{1}, 1-\xi_{1}\right) \in\right] e_{2}, e_{1}[$ such that $\xi_{1} \in\left[\frac{\varepsilon}{1+\varepsilon}, \frac{1}{1+\varepsilon}\right]$ and any $i \in \llbracket \delta N \xi_{1},(1-\delta) N \xi_{1} \rrbracket$,

$$
\mathbb{P}\left(\pi^{0,\lfloor N \xi\rfloor} \cap \mathcal{C}_{i, r}^{\xi}=\varnothing\right) \leq C r^{-3} .
$$

Proof. Abbreviate $p=\left(p_{1}, p_{2}\right)=\lfloor\xi N\rfloor$. The proof shows that with high probability, $\pi^{0, p}$ is captured between two geodesics of stationary LPP processes and then controls the probability that these geodesics deviate from the $\xi$-ray. Figure 4.1 illustrates the proof.

Take $\rho^{\star}=\rho(\xi)+r N^{-\frac{1}{3}}$ and $\rho_{\star}=\rho(\xi)-r N^{-\frac{1}{3}}$ with characteristic directions $\xi^{\star}=\xi\left(\rho^{\star}\right)$ and $\xi_{\star}=\xi\left(\rho_{\star}\right)$. The upper bound on $r$ guarantees that $\rho^{\star}, \rho_{\star} \in\left[\varepsilon^{\prime}, 1-\varepsilon^{\prime}\right]$. Let $v^{\star}$ be the geodesic of $G_{0, p}^{\rho^{\star}}$ and $v_{\star}$ the geodesic of $G_{0, p}^{\rho \star}$. We couple the weights of the three LPP processes as follows. The bulk weights $\left\{\omega_{x}\right\}_{x \in \mathbb{Z}_{>0}^{2}}$ are the same for each LPP process. On the axes, we couple so that, for $i, j \geq 1$,

$$
\omega_{i e_{1}} \leq I_{i e_{1}}^{\rho_{\star}} \wedge I_{i e_{1}}^{\rho^{\star}} \quad \text { and } \quad \omega_{j e_{2}} \leq J_{j e_{2}}^{\rho_{\star}} \wedge J_{j e_{2}}^{\rho^{\star}}
$$

We can use here the coupling of Theorem 3.1(iii). However, the present proof does not need the joint stationarity of the LPP processes with parameters $\rho^{\star}$ and $\rho_{\star}$, and hence a simpler coupling is adequate. In this simpler coupling, we take all the triples $\left(\omega_{i e_{1}}, I_{i e_{1}}^{\rho_{\star}}, I_{i e_{1}}^{\rho^{\star}}\right)$ and $\left(\omega_{j e_{2}}, J_{j e_{2}}^{\rho_{\star}}, J_{j e_{2}}^{\rho^{\star}}\right)$ mutually independent across the indices $i, j$ and then couple within each triple to have the inequalities (4.16) and 
the right marginal distributions

$$
\omega_{x} \sim \operatorname{Exp}(1), \quad I_{i e_{1}}^{\alpha} \sim \operatorname{Exp}(1-\alpha), \quad \text { and } \quad J_{j e_{2}}^{\alpha} \sim \operatorname{Exp}(\alpha)
$$

We develop estimates to control the location of $v_{\star}$. Similar reasoning applies to $v^{\star}$. The mean value theorem applied to the function $\xi_{2} / \xi_{1}=\left(\frac{\rho}{1-\rho}\right)^{2}$ shows that there exist constants $C_{1}(\varepsilon, \delta), C_{2}(\varepsilon, \delta)>0$ such that

$$
C_{1} r N^{\frac{2}{3}} \leq\left(\frac{\xi_{2}}{\xi_{1}}-\frac{\xi_{\star 2}}{\xi_{\star 1}}\right) i \leq C_{2} r N^{\frac{2}{3}} \quad \text { for } \quad i \in \llbracket \delta N, N \rrbracket .
$$

Given $\alpha \in\left[\delta \xi_{1},(1-\delta) \xi_{1}\right]$, define the point $\widetilde{\sigma}=\left(\lfloor\alpha N\rfloor,\left\lfloor\alpha N \xi_{\star 2} / \xi_{\star 1}\right\rfloor\right)$ on the $\xi_{\star}$-ray. Let $G_{\widetilde{\sigma}, p}^{\rho_{\star},[0]}$ be the stationary LPP process on the rectangle $R=\llbracket \widetilde{o}, p \rrbracket$ with boundary weights on the south and west sides given for $i, j \geq 1$ by

$$
I_{\widetilde{o}+i e_{1}}^{\rho_{\star},[0]}=G_{0, \widetilde{o}+i e_{1}}^{\rho_{\star}}-G_{0, \widetilde{o}+(i-1) e_{1}}^{\rho_{\star}} \quad \text { and } \quad J_{\widetilde{o}+j e_{2}}^{\rho_{\star},[0]}=G_{0, \widetilde{o}+j e_{2}}^{\rho_{\star}}-G_{0, \widetilde{o}+(j-1) e_{2}}^{\rho_{\star}} .
$$

Superscript [0] indicates that the boundary weights come from $G_{0, \bullet}^{\rho_{\star}}$. By Lemma B.3, the crossing point of the geodesic $v_{\star}$ through the south and west boundary of $R$ is the exit point of the geodesic of $G_{\widetilde{o}, p}^{\rho_{\star},[0]}$ from that boundary. By (4.17)

$$
\begin{aligned}
\left\{v_{\star} \cap \mathcal{C}_{\lfloor\alpha N\rfloor, 2 C_{2} r}^{\xi}=\varnothing\right\} & \subset\left\{v_{\star} \cap \llbracket \widetilde{o}, \widetilde{o}+2 C_{2} r N^{\frac{2}{3}} e_{2} \rrbracket=\varnothing\right\} \\
& \subset\left\{Z_{\widetilde{o}, p}^{\rho_{\star},[0]} \notin \llbracket-2 C_{2} r N^{\frac{2}{3}}, 0 \rrbracket\right\} .
\end{aligned}
$$

From this,

$$
\begin{aligned}
\mathbb{P}\left(v_{\star} \cap \mathcal{C}_{\lfloor\alpha N\rfloor, 2 C_{2} r}^{\xi}\right. & =\varnothing) \leq \mathbb{P}\left(Z_{\widetilde{\sigma}, p}^{\rho \star} \notin \llbracket-2 C_{2} r N^{\frac{2}{3}}, 0 \rrbracket\right) \\
& =\mathbb{P}\left(Z_{\widetilde{\sigma}, p}^{\rho \star}>0\right)+\mathbb{P}\left(Z_{\widetilde{o}, p}^{\rho \star}<-2 C_{2} r N^{\frac{2}{3}}\right)
\end{aligned}
$$

(The superscript [0] can be dropped from $Z_{\widetilde{o}, p}^{\rho_{\star},[0]}$ in probability statements because it makes no difference to the distribution.) We show that the last two probabilities are small. Let

$$
y=p_{2}-\frac{\xi_{\star 2}}{\xi \star 1} N \xi_{1}
$$

be the vertical distance between the rays $\xi$ and $\xi \star$ along the east boundary of $R$. By (4.17),

$$
C_{1} r N^{\frac{2}{3}} \leq y \leq C_{2} r N^{\frac{2}{3}}
$$

Since $p-y e_{2}-\widetilde{o}$ points in the characteristic direction of $\rho_{\star}$, the bounds below follow from (4.13) and (4.6) for a constant $C=C(\varepsilon, \delta)$, uniformly for $\xi_{1} \in\left[\frac{\varepsilon}{1+\varepsilon}, \frac{1}{1+\varepsilon}\right]$ and $\alpha \in\left[\delta \xi_{1},(1-\delta) \xi_{1}\right]$ :

$$
\mathbb{P}\left(Z_{\widetilde{o}, p}^{\rho \star}>0\right) \leq C r^{-3}
$$

and (with first an application of Lemma B.4)

$$
\begin{aligned}
\mathbb{P}\left(Z_{\widetilde{o}, p}^{\rho_{\star}}<-2 C_{2} r N^{\frac{2}{3}}\right) & =\mathbb{P}\left(Z_{\widetilde{o}, p-\lfloor y\rfloor e_{2}}^{\rho_{\star}}<-2 C_{2} r N^{\frac{2}{3}}+\lfloor y\rfloor\right) \\
& \leq \mathbb{P}\left(Z_{\widetilde{o}, p-\lfloor y\rfloor e_{2}}^{\rho \star}<-C_{2} r N^{\frac{2}{3}}\right) \leq C r^{-3}
\end{aligned}
$$


Substituting this into (4.18) gives a constant $C(\varepsilon, \delta)$ independent of $\xi, \alpha$ such that

$$
\mathbb{P}\left(v_{\star} \cap \mathcal{C}_{\lfloor\alpha N\rfloor, 2 C_{2} r}^{\xi}=\varnothing\right) \leq C r^{-3} .
$$

Similarly, one shows that

$$
\mathbb{P}\left(v^{\star} \cap \mathcal{C}_{\lfloor\alpha N\rfloor, 2 C_{2} r}^{\xi}=\varnothing\right) \leq C r^{-3} .
$$

Combining the bounds above with Corollary 4.3 gives the next estimate, still with a constant $C(\varepsilon, \delta)$ independent of $\xi, \alpha$ :

$$
\mathbb{P}\left\{Z_{0, p}^{\rho_{\star}}<0, Z_{0, p}^{\rho^{\star}}>0, v^{\star} \cap \mathcal{C}_{\lfloor\alpha N\rfloor, 2 C_{2} r}^{\xi} \neq \varnothing, v_{\star} \cap \mathcal{C}_{\lfloor\alpha N\rfloor, 2 C_{2} r}^{\xi} \neq \varnothing\right\} \geq 1-C r^{-3} .
$$

The proof of the lemma is complete once we show that the event above implies the complement of (4.15): namely, that

$$
\begin{aligned}
& \left\{Z_{0, p}^{\rho_{\star}}<0, Z_{0, p}^{\rho^{\star}}>0, v^{\star} \cap \mathcal{C}_{\lfloor\alpha N\rfloor, 2 C_{2} r}^{\xi} \neq \varnothing, v_{\star} \cap \mathcal{C}_{\lfloor\alpha N\rfloor, 2 C_{2} r}^{\xi} \neq \varnothing\right\} \\
& \quad \subset\left\{\pi^{0, p} \cap \mathcal{C}_{\alpha N, 2 C_{2} r}^{\xi} \neq \varnothing\right\} .
\end{aligned}
$$

The inclusion (4.19) holds because conditions $Z_{0, p}^{\rho_{\star}}<0, Z_{0, p}^{\rho^{\star}}>0$ imply that the geodesic $\pi^{0, p}$ runs between geodesics $v_{\star}$ and $v^{\star}$, with $v_{\star}$ above $\pi^{0, p}$ and $v^{\star}$ below and to the right of $\pi^{0, p}$. This is where the coupling (4.16) comes in. We argue one of the two cases: namely,

$$
Z_{0, p}^{\rho^{\star}}>0 \text { implies that } \pi^{0, p} \text { never goes strictly to the right of } v^{\star} .
$$

Let $n=|p|_{1}$ so that the geodesics end at $\pi_{n}^{0, p}=v_{n}^{\star}=p$. Suppose claim (4.20) fails, so that at some index $k, \pi_{k}^{0, p}=v_{k}^{\star}=z$ but $v_{k+1}^{\star}=z+e_{2}$ while $\pi_{k+1}^{0, p}=z+e_{1} \cdot Z_{0, p}^{\rho^{\star}}>0$ implies that $k \geq 1$ and $z+e_{2}$ lies in the bulk $\mathbb{Z}_{>0}$. Since $\pi_{[k+1, n]}$ did not follow the bulk path $v_{[k+1, n]}^{\star}$, the weight of $\pi_{[k+1, n]}$ must be strictly larger than that of $v_{[k+1, n]}^{\star}$. But now the first inequality of (4.16) guarantees that path segment $v_{[k+1, n]}^{\star}$ is also inferior to $\pi_{[k+1, n]}$ for the stationary LPP value $G_{0, p}^{\rho^{\star}}$. Thus the separation did not happen.

\section{No bi-infinite geodesic away from the axes}

This section proves Theorem 2.1. Recall the southwest boundary part $\partial^{N}=(\{-N\} \times \llbracket-N,-\varepsilon N \rrbracket) \cup$ $(\llbracket-N,-\varepsilon N \rrbracket \times\{-N\})$ from (2.1). The parameter $\varepsilon>0$ now stays fixed and hence will be suppressed from some notation. As in (4.2), a point $o=\left(o_{1}, o_{2}\right) \in \partial^{N}$ is associated with its direction vector $\left.\xi(o)=\left(\xi_{1}(o), 1-\xi_{1}(o)\right) \in\right] e_{2}, e_{1}[$ and rate parameter $\rho(o) \in(0,1)$ through the relations

$$
\xi(o)=\left(\frac{o_{1}}{o_{1}+o_{2}}, \frac{o_{2}}{o_{1}+o_{2}}\right)=\left(\frac{(1-\rho(o))^{2}}{(1-\rho(o))^{2}+\rho(o)^{2}}, \frac{\rho(o)^{2}}{(1-\rho(o))^{2}+\rho(o)^{2}}\right)
$$

and

$$
\rho(o)=\frac{\sqrt{\left|o_{2}\right|}}{\sqrt{\left|o_{1}\right|}+\sqrt{\left|o_{2}\right|}}=\frac{\sqrt{1-\xi_{1}(o)}}{\sqrt{\xi_{1}(o)}+\sqrt{1-\xi_{1}(o)}} .
$$

For all $o \in \partial^{N}$, we have the bounds

$$
\xi(o) \in\left[\left(\frac{\varepsilon}{1+\varepsilon}, \frac{1}{1+\varepsilon}\right),\left(\frac{1}{1+\varepsilon}, \frac{\varepsilon}{1+\varepsilon}\right)\right] \quad \text { and } \quad \frac{\sqrt{\varepsilon}}{1+\sqrt{\varepsilon}} \leq \rho(o) \leq \frac{1}{1+\sqrt{\varepsilon}} .
$$




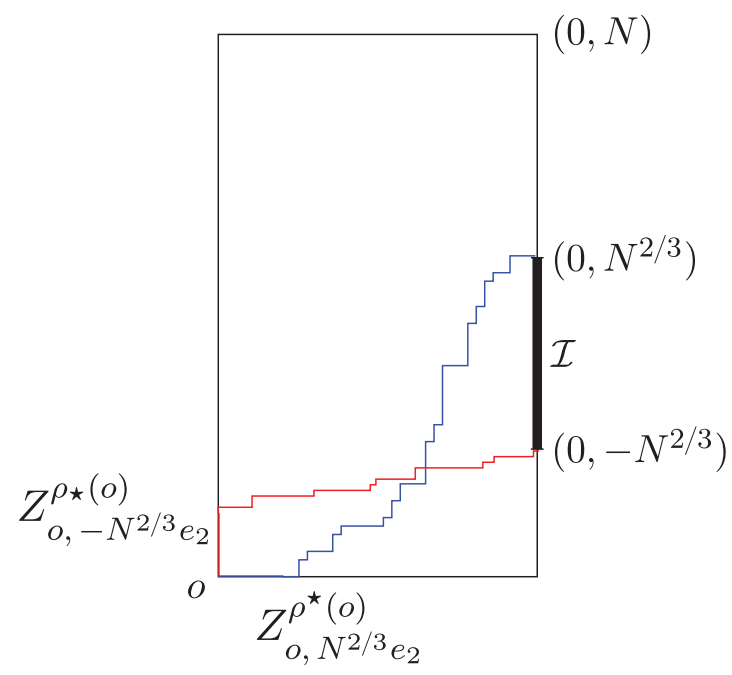

Figure 5.1. Lemma 5.1. For large $r$, the exit point $Z_{o,\left\lfloor N^{2 / 3}\right\rfloor e_{2}}^{\rho^{\star}(o)}$ is far to the right from o on the scale $N^{2 / 3}$. By the uniqueness of finite geodesics, the same holds for $Z_{o, x}^{\rho^{\star}(o)}$ for all $x \in \mathcal{J}$, and similarly for exit points $Z_{o, x}^{\rho_{\star}(o)}$ above $o$.

The proof uses LPP values from points of $\partial^{N}$ to the vertical segment $\mathcal{J}=\{0\} \times \llbracket-N^{\frac{2}{3}}, N^{\frac{2}{3}} \rrbracket$. This latter is indexed by $I=\llbracket-N^{\frac{2}{3}}, N^{\frac{2}{3}} \rrbracket$. For $o \in \partial^{N}$, let

$$
\rho_{\star}(o)=\rho(o)-r N^{-\frac{1}{3}} \quad \text { and } \quad \rho^{\star}(o)=\rho(o)+r N^{-\frac{1}{3}}
$$

and consider the stationary LPP processes $G_{o}^{\rho^{\star}}$, and $G_{o \star}^{\rho_{\star}}$, based at $o$. The next lemma shows that a large enough $r$ forces the exit point of $G_{o, x}^{\rho^{\star}}$ to the $x$-axis and that of $G_{o, x}^{\rho \star}$ to the $y$-axis, arbitrarily far on the $N^{2 / 3}$ scale, with a probability bound that is uniform over $o \in \partial^{N}$ and $x \in \mathcal{J}$. See Figure 5.1 for an illustration.

Lemma 5.1. For $0<\varepsilon<1$, there exist finite positive constants $C_{0}(\varepsilon), C_{1}(\varepsilon)$ such that, whenever $d$ and $r$ satisfy

$$
1 \leq d \leq \frac{1}{2} \varepsilon N^{1 / 3} \quad \text { and } \quad C_{0}(\varepsilon) d \leq r \leq \frac{\sqrt{\varepsilon}}{2(1+\sqrt{\varepsilon})} N^{1 / 3}
$$

the following bounds hold for all $N \geq 1, x \in \mathcal{J}$, and $o \in \partial^{N}$ :

$$
\mathbb{P}\left\{Z_{o, x}^{\rho \star(o)} \geq-d N^{2 / 3}\right\} \leq C_{1}(\varepsilon) r^{-3}
$$

and

$$
\mathbb{P}\left\{Z_{o, x}^{\rho^{\star}(o)} \leq d N^{2 / 3}\right\} \leq C_{1}(\varepsilon) r^{-3},
$$

where $\rho_{\star}(o)=\rho(o)-r N^{-\frac{1}{3}}$ and $\rho^{\star}(o)=\rho(o)+r N^{-\frac{1}{3}}$.

Proof. The upper bound $r \leq \frac{\sqrt{\varepsilon}}{2(1+\sqrt{\varepsilon})} N^{1 / 3}$ guarantees that $\rho_{\star}(o), \rho^{\star}(o) \in\left[\varepsilon^{\prime}, 1-\varepsilon^{\prime}\right]$ for all $\rho(o)$, and hence the estimates from the increment-stationary CGM apply.

We prove (5.5). (5.4) is similar. Represent $o \in \partial^{N, \varepsilon}$ as $o=-(a N, b N)$, where $a \vee b=1$ and $a \wedge b \in[\varepsilon, 1]$. Abbreviate $\rho=\rho(o)$ and $\rho^{\star}=\rho^{\star}(o)$. Then $a / b=\left(\frac{1-\rho}{\rho}\right)^{2}$. 
Uniqueness of geodesics forces the $o$ to $\left\lfloor N^{2 / 3}\right\rfloor e_{2}$ geodesic to stay above the $o$ to $x \in \mathcal{J}$ geodesic. Then apply Lemma B.3 and translate $o$ to the origin 0 to deduce:

$$
\begin{aligned}
\mathbb{P}\left\{Z_{o, x}^{\rho^{\star}} \leq d N^{2 / 3}\right\} & \leq \mathbb{P}\left\{Z_{o,\left\lfloor N^{2 / 3}\right\rfloor e_{2}}^{\rho^{\star}} \leq d N^{2 / 3}\right\}=\mathbb{P}\left\{Z_{o,\left\lfloor N^{2 / 3}\right\rfloor e_{2}-\left\lfloor d N^{2 / 3}\right\rfloor e_{1}}^{\rho^{\star}} \leq-1\right\} \\
& =\mathbb{P}\left\{Z_{0,\left(a N-\left\lfloor d N^{2 / 3}\right\rfloor, b N+\left\lfloor N^{2 / 3}\right\rfloor\right)}^{\rho^{\star}} \leq-1\right\} .
\end{aligned}
$$

Define a new scaling parameter $M$ by

$$
a N-d N^{2 / 3}=M \xi_{1}\left(\rho^{\star}\right) .
$$

The assumption $d \leq \frac{1}{2} \varepsilon N^{1 / 3} \leq \frac{1}{2} a N^{1 / 3}$ guarantees that $M>0$.

To apply (4.14) to the last probability in (5.6), we bound the deviation of $b N+\left\lfloor N^{2 / 3}\right\rfloor$ from the characteristic point $M \xi_{2}\left(\rho^{\star}\right)$.

$$
\begin{aligned}
M \xi_{2}\left(\rho^{\star}\right)-b N-\left\lfloor N^{2 / 3}\right\rfloor \geq M \xi_{2}\left(\rho^{\star}\right)-b N-N^{2 / 3} \\
\quad=\left(a N-d N^{2 / 3}\right)\left(\frac{\rho^{\star}}{1-\rho^{\star}}\right)^{2}-a N\left(\frac{\rho}{1-\rho}\right)^{2}-N^{2 / 3} \\
\quad=N^{2 / 3}\left(\operatorname{ar} \frac{\rho^{\star}+\rho-2 \rho \rho^{\star}}{\left(1-\rho^{\star}\right)^{2}(1-\rho)^{2}}-d\left(\frac{\rho^{\star}}{1-\rho^{\star}}\right)^{2}-1\right) \\
=M^{2 / 3} \cdot \frac{\alpha\left[\rho^{\star}\right]^{2 / 3}\left(1-\rho^{\star}\right)^{4 / 3}}{\left(a-d N^{-1 / 3}\right)^{2 / 3}} \cdot \frac{\operatorname{ar}\left(\rho^{\star}+\rho-2 \rho \rho^{\star}\right)-d\left(\rho^{\star}\right)^{2}(1-\rho)^{2}-\left(1-\rho^{\star}\right)^{2}(1-\rho)^{2}}{\left(1-\rho^{\star}\right)^{2}(1-\rho)^{2}} .
\end{aligned}
$$

The above followed from definitions (4.2) and (4.3). Next bound the last line from below. The assumption $r \leq \frac{\sqrt{\varepsilon}}{2(1+\sqrt{\varepsilon})} N^{1 / 3}$ guarantees that

$$
\rho^{\star}+\rho-2 \rho \rho^{\star} \geq c_{6}(\varepsilon)
$$

for a positive constant $c_{6}(\varepsilon)$ whose precise value is immaterial. Use additionally $\alpha\left[\rho^{\star}\right] \geq 1, a \geq \varepsilon$ and $d \geq 1$ to get the lower bound

$$
M \xi_{2}\left(\rho^{\star}\right)-b N-\left\lfloor N^{2 / 3}\right\rfloor \geq M^{2 / 3}\left(c_{6}(\varepsilon) \varepsilon r-2 d\right) \geq M^{2 / 3} c_{7}(\varepsilon) r
$$

where the last inequality follows from assuming $r \geq 4 d c_{6}(\varepsilon)^{-1} \varepsilon^{-1} \equiv C_{0}(\varepsilon) d$ and defining $c_{7}(\varepsilon)$ suitably. Returning to (5.6), we have

$$
\begin{aligned}
\mathbb{P}\left\{Z_{o, x}^{\rho^{\star}} \leq d N^{2 / 3}\right\} & =\mathbb{P}\left\{Z_{0,\left(a N-\left\lfloor d N^{2 / 3}\right\rfloor, b N+\left\lfloor N^{2 / 3}\right\rfloor\right)}^{\rho^{\star}} \leq-1\right\} \\
& \leq \mathbb{P}\left\{Z_{0,\left(\left\lfloor M \xi_{1}\left(\rho^{\star}\right)\right\rfloor,\left\lfloor M \xi_{2}\left(\rho^{\star}\right)\right\rfloor-\left\lfloor M^{2 / 3} r c_{7}(\varepsilon)\right\rfloor\right)}^{\rho^{\star}} \leq-1\right\} \leq C_{1}(\varepsilon) r^{-3} .
\end{aligned}
$$

The last inequality comes from (4.14). The constant $\kappa$ in (4.14) can be fixed at 2 and ignored.

We introduce a pair of parameters $d=\left(d_{1}, d_{2}\right) \in \mathbb{Z}_{\geq 1}^{2}$ that control coarse graining on the scale $N^{2 / 3}$, $d_{1}$ on the southwest portion of the boundary of the square $\llbracket-N, N \rrbracket^{2}$ and $d_{2}$ on the northeast part. For $o \in \partial^{N}$, let

$$
\mathcal{J}_{o, d}=\left\{u \in \partial^{N}:|u-o|_{1} \leq \frac{1}{2} d_{1} N^{\frac{2}{3}}\right\}
$$

and

$$
o_{c}=\text { the unique minimal point of } \mathcal{J}_{o, d} \text { in the coordinatewise partial order on } \mathbb{Z}^{2} .
$$

For an illustration of $o, o_{c}$ and $\mathcal{J}_{o, d}$, see Figure 5.2. 
For a given point $o \in \partial^{N}$, we compare the LPP processes $G_{u, \bullet}$ from initial points $u \in \mathcal{J}_{o, d}$ with increment-stationary LPP processes $G_{o_{c}}^{\rho_{\star}}, \bullet$ and $G_{o_{c}}^{\rho^{\star}}, \bullet$ with base point $o_{c}$ and parameters

$$
\rho_{\star}=\rho\left(o_{c}\right)-r N^{-\frac{1}{3}} \quad \text { and } \quad \rho^{\star}=\rho\left(o_{c}\right)+r N^{-\frac{1}{3}},
$$

assumed to satisfy $\rho_{\star}, \rho^{\star} \in(0,1)$. The weights on the boundaries with corner at $o_{c}$ are coupled as in Theorem 3.1(iii): for $i, j \geq 1$,

$$
\omega_{o_{c}+i e_{1}} \leq I_{o_{c}+i e_{1}}^{\rho_{\star}} \leq I_{o_{c}+i e_{1}}^{\rho^{\star}} \quad \text { and } \quad \omega_{o_{c}+j e_{2}} \leq J_{o_{c}+j e_{2}}^{\rho^{\star}} \leq J_{o_{c}+j e_{2}}^{\rho_{\star}} .
$$

Associated with these LPP processes are vertical increment variables on the $y$-axis. We are concerned now only with the range $j \in I$, so the increment variables below are well-defined once $-\varepsilon N<-N^{2 / 3}$. For $u \in \mathcal{J}_{o, d}$ and $\rho \in\left\{\rho_{\star}, \rho^{\star}\right\}$, let

$$
J_{j}^{u}=G_{u, j e_{2}}-G_{u,(j-1) e_{2}} \quad \text { and } \quad J_{j}^{\rho}=G_{o_{c}, j e_{2}}^{\rho}-G_{o_{c},(j-1) e_{2}}^{\rho}, \quad j \in I .
$$

Define the event

$$
A_{o, d}=\left\{Z_{o_{c},-\left\lceil N^{2 / 3}\right\rceil e_{2}}^{\rho_{\star}}<-d_{1} N^{\frac{2}{3}}, Z_{o_{c},\left\lceil N^{2 / 3}\right\rceil e_{2}}^{\rho^{\star}}>d_{1} N^{\frac{2}{3}}\right\} .
$$

Lemma 5.2. Let $N \geq N_{0}(\varepsilon)$ so that the increment variables are well-defined for $j \in I$. On the event $A_{o, d}$, we have the inequalities

$$
J_{j}^{\rho^{\star}} \leq J_{j}^{u} \leq J_{j}^{\rho_{\star}} \quad \forall j \in I, u \in \mathcal{J}_{o, d} .
$$

There exist constants $C_{0}(\varepsilon), C_{1}(\varepsilon)$ such that, whenever $\left(d_{1}, r\right)$ satisfy (5.3), then

$$
\mathbb{P}\left(A_{o, d}^{c}\right) \leq C_{1}(\varepsilon) r^{-3} \quad \text { for all } o \in \partial^{N} .
$$

Proof. We prove the second inequality of (5.9). The first one comes analogously.

Let $\widetilde{G}_{x, y}$ be the LPP process on the quadrant $o_{c}+\mathbb{Z}_{\geq 0}^{2}$ that uses weights $\widetilde{\omega}$ defined by $\widetilde{\omega}_{o_{c}+j e_{2}}=J_{o_{c}+j e_{2}}^{\rho_{\star}}$ for $j \geq 1, \widetilde{\omega}_{o_{c}}=0$, and $\widetilde{\omega}_{o_{c}+x}=\omega_{o_{c}+x}$ for $x \cdot e_{1}>0$.

Suppose first that $u=o_{c}+\ell e_{2}$ for some $\ell \geq 0$. The uniqueness of finite geodesics together with the first inequality of the event $A_{o, d}$ in (5.8) implies that $Z_{o_{c}, x}^{\rho_{\star}}<-d_{1} N^{\frac{2}{3}}$ for all $x \in \mathcal{J}$. Hence both $u$ and $u+e_{2}$ lie on the geodesic of $G_{o_{c}, x}^{\rho_{\star}}$ for all $x \in \mathcal{J}$. Consequently,

$$
G_{o_{c}, x+e_{2}}^{\rho \star}-G_{o_{c}, x}^{\rho \star}=\widetilde{G}_{u, x+e_{2}}-\widetilde{G}_{u, x} .
$$

Lemma B.1 gives the inequality

$$
\widetilde{G}_{u, x+e_{2}}-\widetilde{G}_{u, x} \geq G_{u, x+e_{2}}-G_{u, x} .
$$

The other case is that $u=o_{c}+k e_{1}$ for some $k \geq 1$. Then $G_{o_{c}, x}^{\rho_{\star}}=\widetilde{G}_{o_{c}, x}$ and $G_{u, x}=\widetilde{G}_{u, x}$. This time, the conclusion follows from Lemma B.2.

Bound (5.10) comes from Lemma 5.1.

Next we perform the analogous construction in the northeast quadrant. As in (2.2), $\widehat{\partial}^{N}=\{N\} \times$ $\llbracket \varepsilon N, N \rrbracket \cup \llbracket \varepsilon N, N \rrbracket \times\{N\}$. A point $\widehat{o}=\left(\widehat{o}_{1}, \widehat{o}_{2}\right) \in \widehat{\partial}^{N}$ is associated with a density $\rho(\widehat{o}) \in(0,1)$ and a direction $\xi(\widehat{o}) \in] e_{2}, e_{1}$ [ through the relations (5.1)-(5.2). For $x, y \in \mathbb{Z}^{2}$ such that $x \leq y$, define a reversed last-passage process $\widehat{G}_{y, x}=G_{x, y}$ in terms of the i.i.d. $\operatorname{Exp}(1) \omega$-weights.

For each parameter value $\rho \in(0,1)$, analogously with (3.1)-(3.2), we define stationary last-passage percolation processes $\widehat{G}_{\widehat{o}, \bullet}^{\rho}$ on the southwest quadrant $\widehat{o}+\mathbb{Z}_{\leq 0}^{2}$. Let $\left\{\widehat{I}_{\widehat{o}-i e_{1}}^{\rho}\right\}_{i \in \mathbb{Z}_{>0}}$ and $\left\{\widehat{J}_{\widehat{o}-j e_{2}}^{\rho}\right\}_{j \in \mathbb{Z}_{>0}}$ be mutually independent boundary weights on the north and east, with marginal distributions $\widehat{I}_{\widehat{o}-i e_{1}}^{\rho} \sim$ 
$\operatorname{Exp}(1-\rho)$ and $\widehat{J}_{\widehat{o}-j e_{2}}^{\rho} \sim \operatorname{Exp}(\rho)$, independent of the boundary variables $I_{o+i e_{1}}^{\rho}, J_{o+j e_{2}}^{\rho}$ in the southwest quadrant. Put $\widehat{G}_{\widehat{o}, \widehat{o}}^{\rho}=0$ and on the boundaries

$$
\widehat{G}_{\widehat{o}, \widehat{o}-k e_{1}}^{\rho}=\sum_{i=1}^{k} \widehat{I}_{\widehat{o}-i e_{1}}^{\rho} \quad \text { and } \quad \widehat{G}_{\widehat{o}, \widehat{o}-l e_{2}}^{\rho}=\sum_{j=1}^{l} \widehat{J}_{\widehat{o}-j e_{2}}^{\rho} .
$$

Then in the bulk for $x=\left(x_{1}, x_{2}\right) \in \widehat{o}+\mathbb{Z}_{<0}^{2}$,

$$
\widehat{G}_{\widehat{o}, x}^{\rho}=\max _{1 \leq k \leq \widehat{o}_{1}-x_{1}}\left\{\sum_{i=1}^{k} \widehat{I}_{\widehat{o}-i e_{1}}^{\rho}+\widehat{G}_{\widehat{o}-k e_{1}-e_{2}, x}\right\} \bigvee \max _{1 \leq \ell \leq \widehat{o}_{2}-x_{2}}\left\{\sum_{j=1}^{\ell} \widehat{J}_{\widehat{o}-j e_{2}}^{\rho}+\widehat{G}_{\widehat{o}-\ell e_{2}-e_{1}, x}\right\} .
$$

For a southwest endpoint $p \in \widehat{o}+\mathbb{Z}_{<0}^{2}$, let $\widehat{Z}_{\widehat{o}, p}^{\rho}$ be the signed exit point of the geodesic $\widehat{\pi}_{\bullet}^{\rho, \widehat{o}, p}$ of $\widehat{G}_{\widehat{o}, p}^{\rho}$ from the north and east boundaries of $\widehat{o}+\mathbb{Z}_{\leq 0}^{2}$. Precisely,

$$
\widehat{Z}_{\widehat{o}, x}^{\rho}= \begin{cases}\underset{k \geq 1}{\arg \max }\left\{\sum_{i=1}^{k} \widehat{I}_{\widehat{o}-i e_{1}}^{\rho}+\widehat{G}_{\widehat{o}-k e_{1}-e_{2}, x}\right\}, & \text { if } \widehat{\pi}_{1}^{\rho, \widehat{o}, x}=\widehat{o}-e_{1}, \\ -\underset{\ell \geq 1}{\arg \max }\left\{\sum_{j=1}^{\ell} \widehat{J}_{\widehat{o}-j e_{2}}^{\rho}+\widehat{G}_{\widehat{o}-\ell e_{2}-e_{1}, x}\right\}, & \text { if } \widehat{\pi}_{1}^{\rho, \widehat{o}, x}=\widehat{o}-e_{2} .\end{cases}
$$

For $\widehat{o} \in \widehat{\partial}^{N}$, let

$$
\widehat{\mathcal{J}}_{\widehat{o}, d}=\left\{v \in \widehat{\partial}^{N}:|v-\widehat{o}|_{1} \leq \frac{1}{2} d_{2} N^{2 / 3}\right\}
$$

and (with an illustration in Figure 5.2),

$$
\widehat{o}_{c}=\text { the unique maximal point of } \widehat{\mathcal{J}}_{\widehat{o}, d} \text { in the coordinatewise partial order on } \mathbb{Z}^{2} \text {. }
$$

Define again parameters

$$
\rho_{\star}=\rho\left(\widehat{o}_{c}\right)-r N^{-\frac{1}{3}} \quad \text { and } \quad \rho^{\star}=\rho\left(\widehat{o}_{c}\right)+r N^{-\frac{1}{3}} .
$$

The weights on the boundaries with corner at $\widehat{o}_{c}$ are coupled as in Theorem 3.1(iii): for $i, j \geq 1$,

$$
\omega_{\widehat{o}_{c}-i e_{1}} \leq \widehat{I}_{\widehat{o}_{c}-i e_{1}}^{\rho \star} \leq \widehat{I}_{\widehat{o}_{c}-i e_{1}}^{\rho^{\star}} \quad \text { and } \quad \omega_{\widehat{o}_{c}-j e_{2}} \leq \widehat{J}_{\widehat{o}_{c}-j e_{2}}^{\rho^{\star}} \leq \widehat{J}_{\widehat{o}_{c}-j e_{2}}^{\rho \star}
$$

Define increment variables on the vertical edges $\left\{\left(x+e_{1}, x+e_{1}+e_{2}\right): x \in \mathcal{J}\right\}$ shifted by $e_{1}$ from J. For $v \in \widehat{\mathcal{J}}_{\widehat{o}, d}, j \in I$, and $\rho \in\left\{\rho_{\star}, \rho^{\star}\right\}$, let

$$
\widehat{J}_{j}^{v}=\widehat{G}_{v, e_{1}+(j-1) e_{2}}-\widehat{G}_{v, e_{1}+j e_{2}} \quad \text { and } \quad \widehat{J}_{j}^{\rho}=\widehat{G}_{\widehat{o}_{c}, e_{1}+(j-1) e_{2}}^{\rho}-\widehat{G}_{\widehat{o}_{c}, e_{1}+j e_{2}}^{\rho} .
$$

Define the event

$$
B_{\widehat{o}, d}=\left\{\widehat{Z}_{\widehat{o}_{c},\left\lceil N^{2 / 3}\right\rceil e_{2}+e_{1}}^{\rho_{\star}}<-d_{2} N^{\frac{2}{3}}, \widehat{Z}_{\widehat{o}_{c},-\left\lceil N^{2 / 3}\right\rceil e_{2}+e_{1}}^{\rho^{\star}}>d_{2} N^{\frac{2}{3}}\right\} .
$$

We have this analogue of Lemma 5.2.

Lemma 5.3. Let $N \geq N_{0}(\varepsilon)$ so that the increment variables are well-defined for $j \in I$. On the event $B_{\widehat{o}, d}$, we have the inequalities

$$
\widehat{J}_{j}^{\rho^{\star}} \leq \widehat{J}_{j}^{v} \leq \widehat{J}_{j}^{\rho^{\star}} \quad \forall j \in I, v \in \widehat{\mathcal{J}}_{\widehat{o}, d}
$$


There exist constants $C_{0}(\varepsilon), C_{1}(\varepsilon)$ such that, whenever $\left(d_{2}, r\right)$ satisfy (5.3), then

$$
\mathbb{P}\left(B_{\widehat{o}, d}^{c}\right) \leq C_{1}(\varepsilon) r^{-3} \quad \text { for all } \widehat{o} \in \widehat{\partial}^{N} .
$$

Let $o \in \partial^{N}, \widehat{o} \in \widehat{\partial}^{N}$ and consider LPP from points $u \in \mathcal{J}_{o, d}$ to the interval $\mathcal{J}$ on the $y$-axis and reverse LPP from points $v \in \widehat{\mathcal{J}}_{\widehat{o}, d}$ to the shifted interval $e_{1}+\mathcal{J}$. Abbreviate $\lambda^{\star}=\rho^{\star}\left(\widehat{o}_{c}\right), \lambda_{\star}=\rho_{\star}\left(\widehat{o}_{c}\right)$ and $\rho^{\star}=\rho^{\star}\left(o_{c}\right), \rho_{\star}=\rho_{\star}\left(o_{c}\right)$.

A given sequence of steps $\left\{X_{j}\right\}$ defines a two-sided walk $S(X)$ by

$$
S_{n}(X)= \begin{cases}\sum_{j=1}^{n} X_{j} & n \geq 1 \\ 0 & n=0 \\ -\sum_{j=n+1}^{0} X_{j} & n<0 .\end{cases}
$$

Use this notation to define three random walks indexed by the edges $\{((0, j),(1, j)): j \in I\}$ that run along the $y$-axis. The steps are defined by

$$
X_{j}^{u, v}=J_{j}^{u}-\widehat{J}_{j}^{v}, \quad Y_{j}^{\prime}=J_{j}^{\rho_{\star}}-\widehat{J}_{j}^{\lambda^{\star}}, \quad \text { and } \quad Y_{j}=J_{j}^{\rho^{\star}}-\widehat{J}_{j}^{\lambda \star} .
$$

The corresponding walks are denoted by

$$
S^{u, v}=S\left(X^{u, v}\right), \quad S^{\prime}=S\left(Y^{\prime}\right), \quad \text { and } \quad S=S(Y) .
$$

Recall the events defined in (5.8) and (5.14).

Lemma 5.4. The processes

$$
\left\{S_{m}^{\prime}: m \in \llbracket-N^{2 / 3},-1 \rrbracket\right\} \quad \text { and } \quad\left\{S_{n}: n \in \llbracket 1, N^{2 / 3} \rrbracket\right\} \quad \text { are independent. }
$$

On the event $A_{o, d} \cap B_{\widehat{o}, d}$, for all $u \in \mathcal{J}_{o, d}$ and $v \in \widehat{\mathcal{J}}_{\widehat{o}, d}$,

$$
\begin{aligned}
& S_{n} \leq S_{n}^{u, v} \leq S_{n}^{\prime} \quad \text { for } n \in \llbracket 1, N^{2 / 3} \rrbracket \\
& \text { and } \quad S_{n}^{\prime} \leq S_{n}^{u, v} \leq S_{n} \quad \text { for } n \in \llbracket-N^{2 / 3},-1 \rrbracket .
\end{aligned}
$$

Proof. The independence of the stationary LPP processes defined on the southwest and northeast quadrants implies that the processes $\left\{J_{j}^{\rho_{\star}}, J_{j}^{\rho^{\star}}\right\}_{j \in I}$ and $\left\{\widehat{J}_{j}^{\lambda_{\star}}, \widehat{J}_{j}^{\lambda^{\star}}\right\}_{j \in I}$ are independent of each other. Theorem 3.1(i) implies that within these processes, $\left\{J_{j}^{\rho_{\star}}\right\}_{j \leq 0}$ and $\left\{J_{j}^{\rho^{\star}}\right\}_{j \geq 1}$ are independent, as are $\left\{\widehat{J}_{j}^{\lambda \star}\right\}_{j \geq 1}$ and $\left\{\widehat{J}_{j}^{\lambda^{\star}}\right\}_{j \leq 0}$. (Note the switch in the direction of indexing: since the geodesics of $G_{\widehat{o}_{c}}^{\rho}, \bullet$ proceed southwest instead of northeast, application of Theorem 3.1 requires reversal of lattice directions.)

Inequalities (5.17) come from the inequalities (5.9) and (5.15).

Next observe that the walk $S^{u, v}$ controls the edge along which the geodesic $\pi^{u, v}$ steps away from the $y$-axis.

$$
\begin{aligned}
G_{u, v} & =\sup _{u_{2} \leq n \leq v_{2}}\left\{G_{u,(0, n)}+\widehat{G}_{v,(1, n)}\right\} \\
& =\sup _{u_{2} \leq n \leq v_{2}}\left\{G_{u,(0,0)}+\left[G_{u,(0, n)}-G_{u,(0,0)}\right]+\widehat{G}_{v,(1,0)}-\left[\widehat{G}_{v,(1,0)}-\widehat{G}_{v,(1, n)}\right]\right\} \\
& =\sup _{u_{2} \leq n \leq v_{2}}\left\{G_{u,(0,0)}+\widehat{G}_{v,(1,0)}+S_{n}^{u, v}\right\} .
\end{aligned}
$$


In consequence,

for $u \in \mathcal{J}_{o, d}$ and $v \in \widehat{\mathcal{J}}_{\widehat{o}, d}$, the geodesic $\pi^{u, v}$ goes along the edge $((0, j),(1, j))$

if and only if $j=\underset{u_{2} \leq n \leq v_{2}}{\arg \max }\left\{S_{n}^{u, v}\right\}$ :

that is, if and only if the almost surely unique maximum of $S_{n}^{u, v}$ is taken at $n=j$.

Let $o \in \partial^{N}$ and $\widehat{o}=-o \in \widehat{\partial}^{N}$, as in Figure 5.2. Let $o_{c} \in \mathcal{J}_{o, d}$ be defined by (5.7) and $\widehat{o}_{c} \in \widehat{\mathcal{J}}_{\widehat{o}, d}$ by (5.13). We will take $d_{1} \neq d_{2}$, so $\widehat{o}_{c} \neq-o_{c}$ is possible. For $u \in \mathcal{J}_{o, d}$ and $v \in \widehat{\mathcal{J}}_{\widehat{o}, d}$, define the event

$$
U^{u, v}=\left\{\text { geodesic } \pi^{u, v} \text { uses edge }((0,0),(1,0))\right\} .
$$

Lemma 5.5. Let $r=N^{\frac{2}{15}}$ and $d=\left(1, N^{\frac{1}{8}}\right)$. There exist constants $C(\varepsilon), N_{0}(\varepsilon)$ such that for all $o \in \partial^{N}$ and $N \geq N_{0}(\varepsilon)$,

$$
\mathbb{P}\left(\bigcup_{u \in \mathcal{J}_{o, d}, v \in \widehat{\mathcal{J}}_{\widehat{o}, d}} U^{u, v}\right) \leq C(\varepsilon) N^{-2 / 5}
$$

Proof. Fix $o \in \partial^{N}$. For any $u \in \mathcal{J}_{o, d}, v \in \widehat{\mathcal{J}}_{\widehat{o}, d}$, by (5.18),

$$
U^{u, v} \subseteq\left\{\sup _{0<l \leq N^{2 / 3}} S_{l}^{u, v}<0\right\} \cap\left\{\sup _{-N^{2 / 3} \leq l<0} S_{l}^{u, v}<0\right\} .
$$

By Lemma 5.4, on the event $A_{o, d} \cap B_{\widehat{o}, d}$,

$$
\begin{aligned}
& \bigcup_{u \in \mathcal{J}_{o, d}, v \in \widehat{\mathcal{J}}_{\hat{o}, d}}\left\{\sup _{0<l \leq N^{2 / 3}} S_{l}^{u, v}<0\right\} \subseteq\left\{\sup _{0<l \leq N^{2 / 3}} S_{l}<0\right\} \\
& \text { and } \bigcup_{u \in \mathcal{J}_{o, d}, v \in \widehat{\mathcal{J}}_{\widehat{o}, d}}\left\{\sup _{-N^{2 / 3} \leq l<0} S_{l}^{u, v}<0\right\} \subseteq\left\{\sup _{-N^{2 / 3} \leq l<0} S_{l}^{\prime}<0\right\} \text {. }
\end{aligned}
$$

Thus on the event $A_{o, d} \cap B_{\widehat{o}, d}$,

$$
\bigcup_{u \in \mathcal{J}_{o, d}, v \in \widehat{\mathfrak{J}}_{\hat{o}, d}} U^{u, v} \subseteq\left\{\sup _{0<l \leq N^{2 / 3}} S_{l}<0\right\} \cap\left\{\sup _{-N^{2 / 3} \leq l<0} S_{l}^{\prime}<0\right\} .
$$

By the independence claim of Lemma 5.4,

$$
\mathbb{P}\left(\bigcup_{u \in \mathcal{J}_{o, d}, v \in \widehat{\mathcal{J}}_{\widehat{o}, d}} U^{u, v}\right) \leq \mathbb{P}\left(\sup _{0<l \leq N^{2 / 3}} S_{l}<0\right) \mathbb{P}\left(\sup _{-N^{2 / 3} \leq l<0} S_{l}^{\prime}<0\right)+\mathbb{P}\left(A_{o, d}^{c} \cup B_{\widehat{o}, d}^{c}\right) .
$$

Let $\rho=\rho\left(o_{c}\right)$ and $\lambda=\rho\left(\widehat{o}_{c}\right)$. Since $\rho(\widehat{o})=\rho(o)$, there is a constant $C(\varepsilon)$ such that, for $N \geq 1$,

$$
|\rho-\lambda| \leq C(\varepsilon)\left(d_{2}+d_{1}\right) N^{-\frac{1}{3}} \leq C(\varepsilon) N^{-5 / 24} .
$$

Each step of the random walk $S$ on $\llbracket 1, N^{\frac{2}{3}} \rrbracket$ is the difference of independent exponential random variables with parameters $\rho^{\star}=\rho+r N^{-\frac{1}{3}}$ and $\lambda_{\star}=\lambda-r N^{-\frac{1}{3}}$. Similarly, each step of the random walk $S^{\prime}$ on $\llbracket-N^{2 / 3},-1 \rrbracket$ is the difference of independent exponential random variables with parameters $\rho_{\star}=\rho-r N^{-\frac{1}{3}}$ and $\lambda^{\star}=\lambda+r N^{-\frac{1}{3}}$. Take $r=N^{\frac{2}{15}}$. Then for $N \geq N_{0}(\varepsilon)$, we have $\rho^{\star}>\lambda_{\star}$. (By (5.21), 

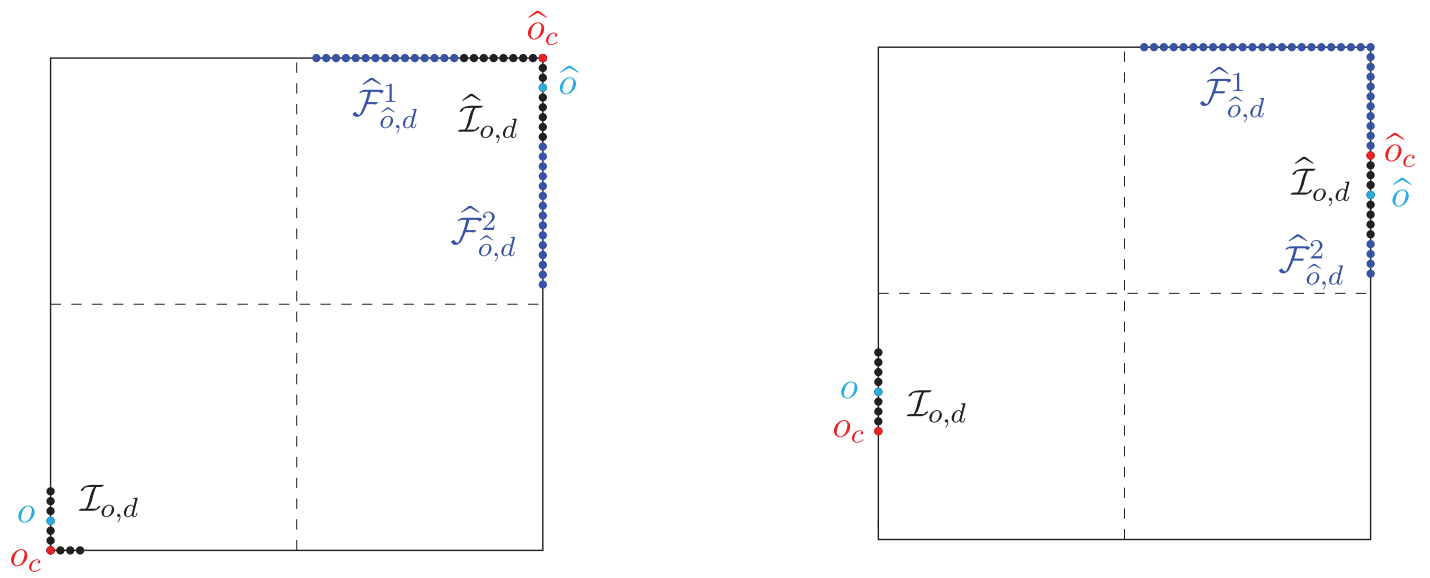

Figure 5.2. The square $\llbracket-N, N \rrbracket^{2}$ with two possible arrangements of the segments $\mathcal{J}_{o, d}$, $\widehat{\mathcal{J}}_{o, d}$ and $\widehat{\mathcal{F}}_{\widehat{o}, d}=\widehat{\mathcal{F}}_{\widehat{o}, d}^{1} \cup \widehat{\mathcal{F}}_{\widehat{o}, d}^{2}$ on the boundary of the square. In both cases, $\widehat{o}=-o$.

we can take $N_{0}(\varepsilon)=C(\varepsilon)^{120}$.) Inequality (C.1) with $\alpha=\rho^{\star}$ and $\beta=\lambda \star$ gives the bound

$$
\begin{aligned}
\mathbb{P}\left(\sup _{0<l \leq N^{2 / 3}} S_{l}<0\right) & \leq \frac{C}{N^{\frac{1}{3}}}\left(1-\left(\frac{\rho-\lambda+2 r N^{-\frac{1}{3}}}{\rho+\lambda}\right)^{2}\right)^{N^{2 / 3}}+\frac{\rho-\lambda+2 r N^{-\frac{1}{3}}}{\rho+r N^{-\frac{1}{3}}} \\
& \leq \frac{C}{N^{\frac{1}{3}}}\left(1-\left(\frac{\rho-\lambda+2 r N^{-\frac{1}{3}}}{\rho+\lambda}\right)^{2}\right)^{N^{2 / 3}}+C\left(d_{1}+d_{2}+r\right) N^{-\frac{1}{3}} .
\end{aligned}
$$

With $r=N^{\frac{2}{15}}$ and $d=\left(d_{1}, d_{2}\right)=\left(1, N^{\frac{1}{8}}\right)$, the last line is dominated by the last term. Thus there is a constant $C_{3}(\varepsilon)>0$ not depending on $o$, such that

$$
\mathbb{P}\left(\sup _{0<l \leq N^{2 / 3}} S_{l}<0\right) \leq C_{3} N^{-\frac{1}{5}} .
$$

Similarly, one shows that

$$
\mathbb{P}\left(\sup _{-N^{2 / 3} \leq l<0} S_{l}^{\prime}<0\right) \leq C_{3} N^{-\frac{1}{5}}
$$

With $r=N^{\frac{2}{15}},(5.10)$ and (5.16) give for $N \geq N_{0}(\varepsilon)$

$$
\mathbb{P}\left(A_{o, d}^{c} \cup B_{\widehat{o}, d}^{c}\right) \leq C r^{-3}=C N^{-\frac{2}{5}} .
$$

To complete the proof, substitute (5.22), (5.23), and (5.24) into (5.20).

Remark 5.6. In the proof above, we can observe where the optimal estimate is lost. Namely, if the probability $\mathbb{P}\left(A_{o, d}^{c} \cup B_{\widehat{o}, d}^{c}\right)$ could be ignored in (5.20), we could take $r$ and $d_{2}$ to be constants. This would result in the bound $C_{3} N^{-1 / 3}$ in (5.22) and (5.23). The end result would be an upper bound of order $N^{-2 / 3}$ on the probability that two opposite blocks of size $N^{2 / 3}$ are connected by a geodesic through the origin. Since geodesics fluctuate on the scale $N^{2 / 3}$, this is the expected order.

Let $o \in \partial^{N}$ and $\widehat{o}=-o$ be as before above Lemma 5.5. For $d=\left(d_{1}, d_{2}\right)$, set

$$
\widehat{\mathcal{F}}_{\widehat{o}, d}=\left\{v \in \widehat{\partial}^{N}:|\widehat{o}-v|_{1}>\frac{1}{2} d_{2} N^{\frac{2}{3}}\right\} .
$$


Lemma 5.7. Let $d=\left(1, N^{\frac{1}{8}}\right)$. There are finite constants $C(\varepsilon)$ and $N_{0}(\varepsilon)$ such that, for any $N \geq N_{0}(\varepsilon)$ and $o \in \partial^{N}$,

$$
\mathbb{P}\left(\bigcup_{u \in \mathcal{J}_{o, d}, v \in \widehat{\mathcal{F}}_{\widehat{o}, d}} U^{u, v}\right) \leq C(\varepsilon) N^{-\frac{3}{8}}
$$

Proof. Define the sets of boundary points

$$
\begin{aligned}
& \partial \widehat{\mathcal{F}}_{\widehat{o}, d}=\left\{v \in \widehat{\mathcal{F}}_{\widehat{o}, d}: \exists u \in \widehat{\mathcal{J}}_{\widehat{o}, d} \text { such that } v \sim u\right\} \\
& \partial \mathcal{J}_{o, d}=\left\{v \in \mathcal{J}_{o, d}: \exists u \in \partial^{N} \backslash \mathcal{J}_{o, d} \text { such that } v \sim u\right\},
\end{aligned}
$$

where $v \sim u$ means that $v$ and $u$ are adjacent in the graph $\mathbb{Z}^{2}$. Their cardinalities satisfy $1 \leq\left|\partial \widehat{\mathcal{F}}_{\widehat{o}, d}\right| \leq$ $\left|\partial \mathcal{J}_{o, d}\right| \leq 2$. (For example, $\partial \widehat{\mathcal{F}}_{\widehat{o}, d}$ is a singleton if $\widehat{\mathcal{J}}_{\widehat{o}, d}$ contains one of the endpoints $(N,\lfloor\varepsilon N\rfloor)$ or $(\lfloor\varepsilon N\rfloor, N)$ of $\widehat{\partial}^{N}$.) We denote the points of $\partial \widehat{\mathcal{F}}_{\widehat{o}, d}$ by $q^{1}, q^{2}$ and those of $\partial \mathcal{J}_{o, d}$ by $h^{1}, h^{2}$, labeled so that these inequalities are satisfied:

$$
\begin{array}{ll}
q_{1}^{1} \leq \widehat{o}_{1} \leq q_{1}^{2}, & q_{2}^{1} \geq \widehat{o}_{2} \geq q_{2}^{2} \\
h_{1}^{1} \geq o_{1} \geq h_{1}^{2}, & h_{2}^{1} \leq o_{2} \leq h_{2}^{2} .
\end{array}
$$

Geometrically, starting from the north pole $(0, N)$ and traversing the boundary of the square $\llbracket-N, N \rrbracket^{2}$ clockwise, we meet the points (those that exist) in this order: $q^{1} \rightarrow \widehat{o} \rightarrow q^{2} \rightarrow h^{1} \rightarrow o \rightarrow h^{2}$ (Figure 5.3).

For points $u \in \partial^{N}, v \in \widehat{\partial}^{N}$ let

$$
\mathcal{P}_{m}^{u, v}=\pi^{u, v} \cap\left\{x \in \mathbb{Z}^{2}: x_{1}=m\right\}
$$

be the intersection of the geodesic $\pi^{u, v}$ with the vertical line at $x_{1}=m$. For $t>0$, let

$$
D_{m, t}^{u, v}=\left\{\inf _{p=\left(p_{1}, p_{2}\right) \in \mathcal{P}_{m}^{u, v}}\left|u_{2}+\frac{v_{2}-u_{2}}{v_{1}-u_{1}}\left(m-u_{1}\right)-p_{2}\right|>t\right\}
$$

be the event that along this vertical line, the geodesic $\pi^{u, v}$ deviates by distance at least $t$ from the straight line segment from $u$ to $v$. We now show that the event in (5.25) implies that one of the geodesics $\pi^{h^{i}, q^{i}}$ deviates by at least order $d_{2} N^{2 / 3}$ from the straight line segment $\left[h^{i}, q^{i}\right]$.

For $o \in \partial^{N}, u \in \partial \mathcal{J}_{o, d}$ and $v \in \partial \widehat{\mathcal{F}}_{\widehat{o}, d}$ decompose as $u=o+e^{u}$ and $v=\widehat{o}+e^{v}$. These vectors satisfy

$$
\left|e^{u}\right|_{1} \leq \frac{1}{2} d_{1} N^{\frac{2}{3}}, \quad\left|e^{v}\right|_{1} \geq \frac{1}{2} d_{2} N^{\frac{2}{3}}, \quad\left|e_{1}^{v}\right| \vee\left|e_{2}^{v}\right| \leq 2(1-\varepsilon) N, \quad \text { and } \quad e_{1}^{v} e_{2}^{v} \leq 0 .
$$

$\widehat{\mathcal{F}}_{\widehat{o}, d}$ is the union of two disjoint pieces separated by $\widehat{\mathcal{J}}_{\widehat{o}, d}$, one of which can be empty. $\widehat{\mathcal{F}}_{\widehat{o}, d}^{1}$ is to the left and above $\widehat{\mathcal{J}}_{\widehat{o}, d}$, separated from $\widehat{\mathcal{J}}_{\widehat{o}, d}$ by the point $q^{1}$. $\widehat{\mathcal{F}}_{\widehat{o}, d}^{2}$ is to the right and below $\widehat{\mathcal{J}}_{\widehat{o}, d}$, separated from $\widehat{\mathcal{J}}_{\widehat{o}, d}$ by the point $q^{2}$. They can be expressed as follows:

$$
\widehat{\mathcal{F}}_{\widehat{o}, d}^{1}=\left\{v \in \widehat{\mathcal{F}}_{\widehat{o}, d}: e_{1}^{v} \leq 0 \leq e_{2}^{v}\right\} \quad \text { and } \quad \widehat{\mathcal{F}}_{\widehat{o}, d}^{2}=\left\{v \in \widehat{\mathcal{F}}_{\widehat{o}, d}: e_{2}^{v} \leq 0 \leq e_{1}^{v}\right\} .
$$

Decompose the point appearing in (5.26) suitably, using $v_{i}-u_{i}=\widehat{o}_{i}+e_{i}^{v}-\left(o_{i}+e_{i}^{u}\right)=-2 o_{i}+e_{i}^{v}-e_{i}^{u}$.

$$
\begin{aligned}
u_{2}+\frac{v_{2}-u_{2}}{v_{1}-u_{1}}\left(m-u_{1}\right) & =o_{2}-\frac{v_{2}-u_{2}}{v_{1}-u_{1}} o_{1}+e_{2}^{u}+\frac{v_{2}-u_{2}}{v_{1}-u_{1}}\left(m-e_{1}^{u}\right) \\
& =\frac{o_{2} e_{1}^{v}-o_{1} e_{2}^{v}}{v_{1}-u_{1}}-\frac{o_{2} e_{1}^{u}-o_{1} e_{2}^{u}}{v_{1}-u_{1}}+e_{2}^{u}+\frac{v_{2}-u_{2}}{v_{1}-u_{1}}\left(m-e_{1}^{u}\right) .
\end{aligned}
$$


The first term on the last line is of order $\Theta\left(d_{2} N^{2 / 3}\right)$ because there is no cancellation in the numerator. It is positive if $v \in \widehat{\mathcal{F}}_{\widehat{o}, d}^{1}$ and negative if $v \in \widehat{\mathcal{F}}_{\widehat{o}, d}^{2}$. This term dominates because $d_{2}>>d_{1}$.

From the calculation above, we bound signed vertical distances from the $x$-axis to the line segment $[u, v]$. In addition to (5.27), we utilize $-N \leq o_{i} \leq-\varepsilon N, 2 N \varepsilon \leq v_{i}-u_{i} \leq 2 N$, and the slope bound $\varepsilon \leq \frac{v_{2}-u_{2}}{v_{1}-u_{1}} \leq \varepsilon^{-1}$.

First, for $u \in \mathcal{J}_{o, d}$ and $v \in \widehat{\mathcal{F}}_{\widehat{o}, d}^{1}$, we bound below the positive distance from the origin to [u,v], so we take $m=0$. The $e^{u}$-terms on line (5.28) are collected together into a single error term.

$$
\begin{aligned}
u_{2}+\frac{v_{2}-u_{2}}{v_{1}-u_{1}}\left(-u_{1}\right) & \geq \frac{\varepsilon N\left|e^{v}\right|_{1}}{2 N}-\left(\frac{N}{2 N \varepsilon}+1+\varepsilon^{-1}\right)\left|e^{u}\right|_{1} \\
& \geq \frac{1}{4} \varepsilon d_{2} N^{\frac{2}{3}}-2 \varepsilon^{-1} d_{1} N^{\frac{2}{3}} \geq \frac{1}{8} \varepsilon d_{2} N^{\frac{2}{3}}
\end{aligned}
$$

In the last inequality, we used $\left(d_{1}, d_{2}\right)=\left(1, N^{1 / 8}\right)$ and took $N \geq\left(16 \varepsilon^{-2}\right)^{8}$.

For $u \in \mathcal{J}_{o, d}$ and $v \in \widehat{\mathcal{F}}_{\widehat{o}, d}^{2}$, we bound above the negative distance from the point $(1,0)$ to $[u, v]$ and hence take $m=1$ :

$$
\begin{aligned}
u_{2}+\frac{v_{2}-u_{2}}{v_{1}-u_{1}}\left(1-u_{1}\right) & \leq-\frac{\varepsilon N\left|e^{v}\right|_{1}}{2 N}+\left(\frac{N}{2 N \varepsilon}+1+\varepsilon^{-1}\right)\left|e^{u}\right|_{1}+\varepsilon^{-1} \\
& \leq-\frac{1}{4} \varepsilon d_{2} N^{\frac{2}{3}}+3 \varepsilon^{-1} d_{1} N^{\frac{2}{3}} \leq-\frac{1}{8} \varepsilon d_{2} N^{\frac{2}{3}}
\end{aligned}
$$

Now suppose that for some $u \in \mathcal{J}_{o, d}$ and $v \in \widehat{\mathcal{F}}_{\widehat{o}, d}$, the geodesic $\pi^{u, v}$ goes through the edge $((0,0),(1,0))$. We have two cases:

(i) If $v \in \widehat{\mathcal{F}}_{\widehat{o}, d}^{1}$, then the geodesic $\pi^{h^{1}, q^{1}}$ stays below and to the right of $\pi^{u, v}$ because both its endpoints are below and to the right of the endpoints of $\pi^{u, v}$. Then (5.29) with $u=h^{1}$ and $v=q^{1}$ shows that at $x$-coordinate $x=0$, the geodesic $\pi^{h^{1}, q^{1}}$ deviates from the straight line segment $\left[h^{1}, q^{1}\right]$ by at least $\frac{1}{8} \varepsilon d_{2} N^{\frac{2}{3}}$. This case is illustrated in Figure 5.3.

(ii) If $v \in \widehat{\mathcal{F}}_{\widehat{o}, d}^{2}$, then the geodesic $\pi^{h^{2}, q^{2}}$ stays above and to the left of $\pi^{u, v}$. Now (5.30) with $u=h^{2}$ and $v=q^{2}$ shows that at $x$-coordinate $x=1$, the geodesic $\pi^{h^{2}, q^{2}}$ deviates from the straight line segment $\left[h^{2}, q^{2}\right]$ by at least $\frac{1}{8} \varepsilon d_{2} N^{\frac{2}{3}}$.

Put cases (i) and (ii) together, and apply Lemma 4.4:

$$
\mathbb{P}\left(\bigcup_{u \in \mathcal{J}_{o, d}, v \in \widehat{\mathcal{F}}_{\widehat{o}, d}} U^{u, v}\right) \leq \mathbb{P}\left(D_{0, C_{1}(\varepsilon) d_{2} N^{2 / 3}}^{h^{1}, q^{1}} \cup D_{1, C_{1}(\varepsilon) d_{2} N^{2 / 3}}^{h^{2}, q^{2}}\right) \leq C(\varepsilon) d_{2}^{-3}=C(\varepsilon) N^{-\frac{3}{8}}
$$

The proof is complete.

Lemma 5.8. There is a constant $C(\varepsilon)$ such that for any $o \in \partial^{N}$,

$$
\mathbb{P}\left(\bigcup_{u \in \mathcal{J}_{o, d}, v \in \widehat{\partial}^{N}} U^{u, v}\right) \leq C(\varepsilon) N^{-\frac{3}{8}}
$$

Proof. Since $\widehat{\partial}^{N}=\widehat{\mathcal{J}}_{\widehat{o}, d} \cup \widehat{\mathcal{F}}_{\widehat{o}, d}$,

$$
\mathbb{P}\left(\bigcup_{u \in \mathcal{J}_{o, d}, v \in \widehat{\partial}^{N}} U^{u, v}\right) \leq \mathbb{P}\left(\bigcup_{u \in \mathcal{J}_{o, d}, v \in \widehat{\mathcal{F}}_{\widehat{o}, d}} U^{u, v}\right)+\mathbb{P}\left(\bigcup_{u \in \mathcal{J}_{o, d}, v \in \widehat{\mathcal{J}}_{\widehat{o}, d}} U^{u, v}\right)
$$

and Lemmas 5.5 and 5.7 give the claimed bound. 


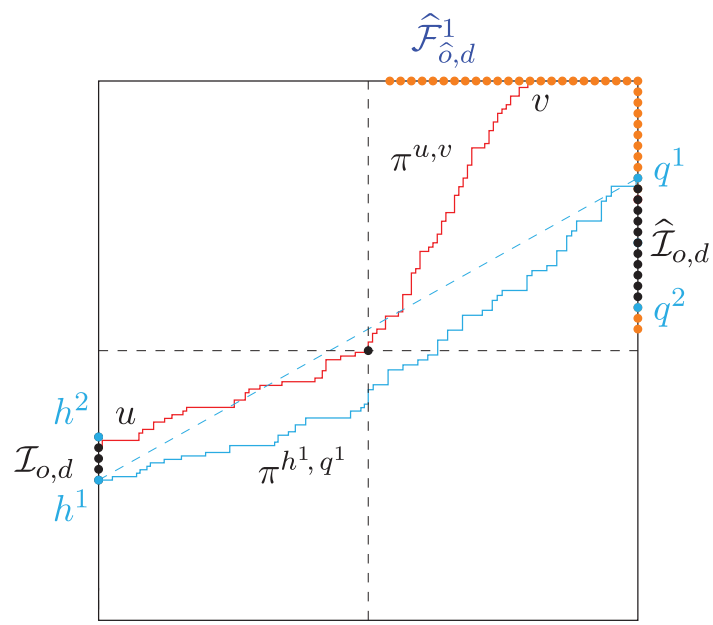

Figure 5.3. Case $(i)$ in the proof of Lemma 5.7. The geodesic $\pi^{u, v}$ connects $\mathcal{J}_{o, d}$ and $\widehat{\mathcal{F}}_{\widehat{o}, d}^{1}$ through the edge $((0,0),(1,0))$. The geodesic $\pi^{h^{1}, q^{1}}$ lies below $\pi^{u, v}$ and hence well below the $\left[h^{1}, q^{1}\right]$ line segment (dashed line).

We come to the final step of the proof that geodesics that connect $\partial^{N}$ and $\widehat{\partial}^{N}$ through the origin are rare. Recall the event $W_{N, \varepsilon}$ defined in (2.3).

Proof of Theorem 2.1. A geodesic through the origin takes after that either an $e_{1}$ or an $e_{2}$ step. By symmetry, it suffices to control only one case. We prove

$$
\mathbb{P}\left(\bigcup_{u \in \partial^{N}, v \in \widehat{\partial}^{N}} U^{u, v}\right) \leq C(\varepsilon) N^{-\frac{1}{24}}
$$

for the event $U^{u, v}$ defined in (5.19). As before, $d=\left(1, N^{\frac{1}{8}}\right)$. To coarse grain $\partial^{N}$ let

$$
\mathcal{O}^{N}=\partial^{N} \cap\left(\left\{\left(-N+i d_{1}\left\lfloor N^{\frac{2}{3}}\right\rfloor,-N\right)\right\}_{i \in \mathbb{Z}_{\geq 0}} \bigcup\left\{\left(-N,-N+j d_{1}\left\lfloor N^{\frac{2}{3}}\right\rfloor\right)\right\}_{j \in \mathbb{Z}_{\geq 0}}\right) .
$$

Then decompose

$$
\bigcup_{u \in \partial^{N}, v \in \widehat{\partial}^{N}} U^{u, v} \subseteq \bigcup_{o \in \mathcal{O}^{N}} \bigcup_{u \in \mathcal{J}_{o, d}, v \in \widehat{\partial}^{N}} U^{u, v}
$$

As $\left|\mathcal{O}^{N}\right| \leq C(\varepsilon) d_{1}^{-1} N^{1-\frac{2}{3}}=C(\varepsilon) N^{\frac{1}{3}}$, a union bound and Lemma 5.8 give (5.31):

$$
\mathbb{P}\left(\bigcup_{u \in \partial^{N}, v \in \widehat{\partial}^{N}} U^{u, v}\right) \leq \sum_{o \in \mathcal{O}^{N}} \mathbb{P}\left(\bigcup_{u \in \mathcal{J}_{o, d}, v \in \widehat{\partial}^{N}} U^{u, v}\right) \leq C(\varepsilon) N^{\frac{1}{3}} N^{-\frac{3}{8}}=C(\varepsilon) N^{-\frac{1}{24}} .
$$

\section{No nontrivial axis-directed geodesic}

First we complete the proof of Theorem 2.2 with the lemma below and then prove the lemma.

Lemma 6.1. Let $\left.\eta_{k}=\left(\eta_{k, 1}, 1-\eta_{k, 1}\right) \in\right] e_{2}, e_{1}[$ be a monotone sequence of directions such that $\eta_{1,1}<\eta_{2,1}<\cdots<\eta_{k, 1}<\cdots$ and $\lim _{k \rightarrow \infty} \eta_{k}=e_{1}$. Let $w_{n, k}=w(n, k)=\left(\left\lfloor n \eta_{k, 1}\right\rfloor, n-\left\lfloor n \eta_{k, 1}\right\rfloor\right) \in \mathbb{Z}_{>0}^{2}$ 
be lattice points such that $\lim _{n \rightarrow \infty} n^{-1} w_{n, k}=\eta_{k}$ for each $k$. Then

$$
\varlimsup_{k \rightarrow \infty} \varlimsup_{n \rightarrow \infty}\left[G_{0, w(n, k)}-G_{e_{2}, w(n, k)}\right]=\infty \quad \mathbb{P} \text {-almost surely. }
$$

Proof of Theorem 2.2. It is enough to prove the case $e_{1}$ for $x=0$.

Fix $\eta_{k}$ and $w_{n, k}$ as in Lemma 6.1, and let $\Omega_{0}$ be the event of full probability on which (6.1) holds. Fix $\omega \in \Omega_{0}$, and suppose that at this $\omega$, there is a semi-infinite geodesic $\pi=\left\{\pi_{n}\right\}_{n \in \mathbb{Z}_{\geq 0}}$ such that $\pi_{0}=0$, $\pi_{\ell}=(\ell-1,1)$ for some $\ell \geq 1$, and $\underline{\lim }_{n \rightarrow \infty} n^{-1} \pi_{n} \cdot e_{2}=0$. We derive a contradiction.

By connecting $e_{2}=(0,1)$ to the point $\pi_{\ell}=(\ell-1,1)$ (now fixed) with a horizontal path, we get the lower bound

$$
G_{e_{2}, \pi_{n}} \geq \sum_{i=0}^{\ell-1} \omega_{(i, 1)}+G_{\pi_{\ell+1}, \pi_{n}} \text { for } n>\ell .
$$

That $\pi$ is a geodesic from $\pi_{0}=0$ implies $G_{0, \pi_{n}}=G_{0, \pi_{\ell}}+G_{\pi_{\ell+1}, \pi_{n}}$ for $n>\ell$. Thus

$$
G_{0, \pi_{n}}-G_{e_{2}, \pi_{n}} \leq G_{0, \pi_{\ell}}-\sum_{i=0}^{\ell-1} \omega_{(i, 1)} \text { for all } n>\ell .
$$

By the assumptions $\underline{\lim } n^{-1} \pi_{n} \cdot e_{2}=0$ and $\left.\eta_{k} \in\right] e_{2}, e_{1}[$, and by the crossing lemma, for each $k$ there are infinitely many indices $n$ such that

$$
G_{0, \pi_{n}}-G_{e_{2}, \pi_{n}} \geq G_{0, w_{n, k}}-G_{e_{2}, w_{n, k}} .
$$

Hence, for each $k$,

$$
\varlimsup_{n \rightarrow \infty}\left[G_{0, \pi_{n}}-G_{e_{2}, \pi_{n}}\right] \geq \varlimsup_{n \rightarrow \infty}\left[G_{0, w_{n, k}}-G_{e_{2}, w_{n, k}}\right] .
$$

Limit (6.1) now contradicts (6.2) because the right-hand side of (6.2) is fixed and finite.

Proof of Lemma 6.1. Let $r<\infty$, and begin by bounding as follows:

$$
\mathbb{P}\left\{\varlimsup_{k \rightarrow \infty} \varlimsup_{n \rightarrow \infty}\left[G_{0, w(n, k)}-G_{e_{2}, w(n, k)}\right] \geq r\right\} \geq \varlimsup_{k \rightarrow \infty} \mathbb{P}\left\{\varlimsup_{n \rightarrow \infty}\left[G_{0, w(n, k)}-G_{e_{2}, w(n, k)}\right]>r\right\} .
$$

We show that the last probability converges to one as $k \rightarrow \infty$.

Choose parameters $\lambda_{k}$ so that

$$
1>\lambda_{k}>\rho\left(\eta_{k}\right)=\frac{\sqrt{1-\eta_{k, 1}}}{\sqrt{1-\eta_{k, 1}}+\sqrt{\eta_{k, 1}}} .
$$

Define the reverse stationary LPP processes $\widehat{G}_{w(n, k), x}^{\lambda_{k}}$ for $x \in w_{n, k}+\mathbb{Z}_{<0}^{2}$ as in (5.11)-(5.12), with parameter $\lambda_{k}$ and northeast base point $w_{n, k}$. As before, for $x \in w_{n, k}+\mathbb{Z}_{<0}^{2}$, let

$$
\widehat{J}_{w(n, k), x}^{\lambda_{k}}=\widehat{G}_{w(n, k), x}^{\lambda_{k}}-\widehat{G}_{w(n, k), x+e_{2}}^{\lambda_{k}}
$$

denote vertical increment variables with distribution $\widehat{J}_{w(n, k), x}^{\lambda_{k}} \sim \operatorname{Exp}\left(\lambda_{k}\right)$. Similarly to the argument in Lemma 5.3, when the geodesic of $\widehat{G}_{w(n, k), 0}^{\lambda_{k}}$ takes a $-e_{1}$ step from $w_{n, k}$, that is, $\widehat{Z}_{w(n, k), 0}^{\lambda_{k}}>0$, the increments satisfy

$$
\widehat{J}_{w(n, k), 0}^{\lambda_{k}} \leq \widehat{G}_{w(n, k), 0}-\widehat{G}_{w(n, k), e_{2}}=G_{0, w(n, k)}-G_{e_{2}, w(n, k)} .
$$

The inequality follows from a combination of Lemmas B.1 and B.2. 


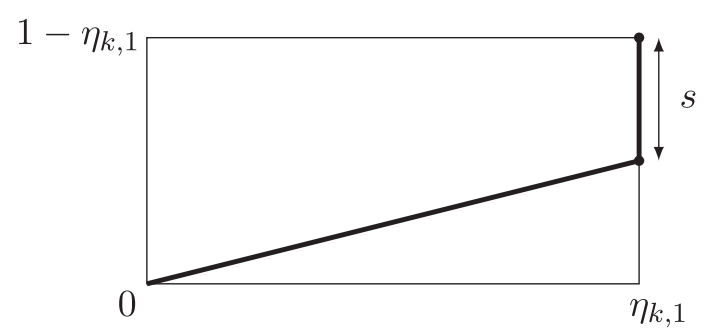

Figure 6.1. When the geodesic is forced to go downward from the northeast corner, the geodesic chooses the distance $s$ on the east side to maximize the sum of $\operatorname{Exp}\left(\lambda_{k}\right)$ weights on the east side and the bulk $L P P$ value between the origin and the point $\left(\eta_{k, 1}, 1-\eta_{k, 1}-s\right)$.

To take advantage of this, we record the limiting shape functions. The stationary LPP process almost surely satisfies

$$
\lim _{n \rightarrow \infty} n^{-1} \widehat{G}_{w(n, k), 0}^{\lambda_{k}}=\frac{\eta_{k, 1}}{1-\lambda_{k}}+\frac{1-\eta_{k, 1}}{\lambda_{k}}
$$

Let $\widehat{G}_{w(n, k), 0}^{\lambda_{k}}\left[\widehat{Z}_{w(n, k), 0}^{\lambda_{k}}<0\right]$ denote the last-passage value computed by maximizing over only those paths that satisfy the condition $\widehat{Z}_{w(n, k), 0}^{\lambda_{k}}<0$ or, equivalently, take first a $-e_{2}$ step from $w_{n, k}$. The limit can be calculated from a macroscopic variational formula (see Figure 6.1 for justification):

$$
\begin{aligned}
\lim _{n \rightarrow \infty} n^{-1} \widehat{G}_{w(n, k), 0}^{\lambda_{k}}\left[\widehat{Z}_{w(n, k), 0}^{\lambda_{k}}<0\right] & =\sup _{0 \leq s \leq 1-\eta_{k, 1}}\left\{\frac{s}{\lambda_{k}}+g\left(\eta_{k, 1}, 1-\eta_{k, 1}-s\right)\right\} \\
& =g\left(\eta_{k, 1}, 1-\eta_{k, 1}\right) .
\end{aligned}
$$

That the supremum is achieved at $s=0$ is a consequence of (6.4). Increasing $\lambda_{k}$ strictly above the characteristic value $\rho\left(\eta_{k}\right)$ as in (6.4) has the effect that the geodesic of $\widehat{G}_{w(n, k), 0}^{\lambda_{k}}$ spends a macroscopic distance on the horizontal boundary $w_{n, k}+\mathbb{Z}_{<0} e_{1}$. Hence, forcing the $-e_{2}$ step from the corner $w_{n, k}$ is suboptimal, and it can be checked directly that

$$
\frac{\eta_{k, 1}}{1-\lambda_{k}}+\frac{1-\eta_{k, 1}}{\lambda_{k}}>g\left(\eta_{k, 1}, 1-\eta_{k, 1}\right) .
$$

We deduce a probability bound from (6.5).

$$
\begin{gathered}
\mathbb{P}\left(G_{0, w(n, k)}-G_{e_{2}, w(n, k)} \leq r\right) \leq \mathbb{P}\left(\widehat{Z}_{w(n, k), 0}^{\lambda_{k}}<0\right)+\mathbb{P}\left(\widehat{J}_{w(n, k), 0}^{\lambda_{k}} \leq r\right) \\
=\mathbb{P}\left\{\widehat{G}_{w(n, k), 0}^{\lambda_{k}}=\widehat{G}_{w(n, k), 0}^{\lambda_{k}}\left[\widehat{Z}_{w(n, k), 0}^{\lambda_{k}}<0\right]\right\}+1-e^{-\lambda_{k} r} .
\end{gathered}
$$

By (6.6), (6.7), and (6.8), the first probability on the last line vanishes as $n \rightarrow \infty$. Switch to complements to get

$$
\underset{n \rightarrow \infty}{\lim _{n \rightarrow \infty}} \mathbb{P}\left(G_{0, w(n, k)}-G_{e_{2}, w(n, k)}>r\right) \geq e^{-\lambda_{k} r} .
$$

From this, upon replacing $r$ by $r+\varepsilon$ for $\varepsilon>0$,

$$
\begin{aligned}
& \mathbb{P}\left\{\varlimsup_{n \rightarrow \infty}\left[G_{0, w(n, k)}-G_{e_{2}, w(n, k)}\right]>r\right\} \\
& \quad \geq \mathbb{P}\left\{G_{0, w(n, k)}-G_{e_{2}, w(n, k)}>r+\varepsilon \text { for infinitely many } n\right\} \geq e^{-\lambda_{k} r-\lambda_{k} \varepsilon} .
\end{aligned}
$$

By assumption, $\eta_{k, 1} \rightarrow 1$. Hence we can satisfy (6.4) while also having $\lambda_{k} \rightarrow 0$. Thus the lower bound in (6.3) equals one. 


\section{Appendix A. Queues}

We formulate last-passage percolation over a bi-infinite strip as a queueing operator. The inputs are two bi-infinite sequences: the inter-arrival process $\mathbf{a}=\left(a_{j}\right)_{j \in \mathbb{Z}}$ and the service process $\mathbf{S}=\left(s_{j}\right)_{j \in \mathbb{Z}}$. The queueing interpretation is that $a_{j}$ is the time between the arrivals of customers $j-1$ and $j$, and $s_{j}$ is the service time of customer $j$. The operations below are well-defined as long as $\lim _{m \rightarrow-\infty} \sum_{i=m}^{0}$ $\left(s_{i}-a_{i+1}\right)=-\infty$.

From inputs $(\mathbf{a}, \mathbf{s})$, three output sequences

$$
\mathbf{d}=D(\mathbf{a}, \mathbf{s}), \quad \mathbf{t}=S(\mathbf{a}, \mathbf{s}), \quad \text { and } \quad \check{\mathbf{s}}=R(\mathbf{a}, \mathbf{s})
$$

are constructed through explicit mappings: the inter-departure process $\mathbf{d}=\left(d_{j}\right)_{j \in \mathbb{Z}}$, the sojourn

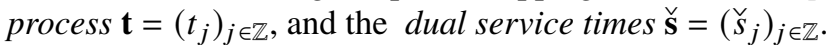

The formulas are as follows. Choose a sequence $G=\left(G_{j}\right)_{j \in \mathbb{Z}}$ that satisfies $a_{j}=G_{j}-G_{j-1}$. Define the sequence $\widetilde{G}=\left(\widetilde{G}_{j}\right)_{j \in \mathbb{Z}}$ by

$$
\widetilde{G}_{j}=\sup _{k: k \leq j}\left\{G_{k}+\sum_{i=k}^{j} s_{i}\right\} .
$$

The supremum above is taken at some finite $k$. Then set

$$
d_{j}=\widetilde{G}_{j}-\widetilde{G}_{j-1}, \quad t_{j}=\widetilde{G}_{j}-G_{j}, \quad \text { and } \quad \check{s}_{j}=a_{j} \wedge t_{j-1} .
$$

The outputs (A.3) do not depend on the choice of $G$ as long as $a_{j}=G_{j}-G_{j-1}$. Letting $k$ be a maximizer for $\widetilde{G}_{j-1}$ in (A.2), we obtain the inequality

$$
d_{j}=\widetilde{G}_{j}-\widetilde{G}_{j-1} \geq\left(G_{k}+\sum_{i=k}^{j} s_{i}\right)-\left(G_{k}+\sum_{i=k}^{j-1} s_{i}\right)=s_{j}
$$

If we start with two coordinatewise ordered inter-arrival processes $a_{j} \leq a_{j}^{\prime}$ (for all $j$ ) and use the same service process $\mathbf{s}$ to compute sojourn processes $\mathbf{t}=S(\mathbf{a}, \mathbf{s})$ and $\mathbf{t}^{\prime}=S\left(\mathbf{a}^{\prime}, \mathbf{s}\right)$, the inequality is reversed:

$$
t_{j}^{\prime}=\widetilde{G}_{j}^{\prime}-G_{j}^{\prime}=\sup _{k: k \leq j}\left\{G_{k}^{\prime}-G_{j}^{\prime}+\sum_{i=k}^{j} s_{i}\right\} \leq \sup _{k: k \leq j}\left\{G_{k}-G_{j}+\sum_{i=k}^{j} s_{i}\right\}=t_{j} .
$$

Note that to compute $\left\{d_{j}, t_{j}, \breve{s}_{j}: j \leq m\right\}$, only inputs $\left\{a_{j}, s_{j}: j \leq m\right\}$ are needed.

The next lemma is a deterministic property of the mappings.

Lemma A.1. The identity $D(D(\mathbf{b}, \mathbf{a}), \mathbf{s})=D(D(\mathbf{b}, R(\mathbf{a}, \mathbf{s})), D(\mathbf{a}, \mathbf{s}))$ holds whenever the sequences $\mathbf{a}, \mathbf{b}, \mathbf{s}$ are such that the operations are well-defined.

Proof. Choose $\left(A_{j}\right)$ and $\left(B_{j}\right)$ so that $A_{j}-A_{j-1}=a_{j}$ and $B_{j}-B_{j-1}=b_{j}$. Then the output of $D(\mathbf{b}, \mathbf{a})$ is the increment sequence of

$$
\widetilde{B}_{\ell}=\sup _{k \leq \ell}\left\{B_{k}+\sum_{i=k}^{\ell} a_{i}\right\}
$$

Next, the output of $D(D(\mathbf{b}, \mathbf{a}), \mathbf{s})$ is the increment sequence of

$$
H_{m}=\sup _{\ell \leq m}\left\{\widetilde{B}_{\ell}+\sum_{j=\ell}^{m} s_{j}\right\}=\sup _{k \leq m}\left\{B_{k}+\max _{\ell: k \leq \ell \leq m}\left[\sum_{i=k}^{\ell} a_{i}+\sum_{j=\ell}^{m} s_{j}\right]\right\} .
$$


Similarly, define first

$$
\widetilde{A}_{j}=\sup _{k: k \leq j}\left\{A_{k}+\sum_{i=k}^{j} s_{i}\right\} \quad \text { and } \quad \check{B}_{\ell}=\sup _{k \leq \ell}\left\{B_{k}+\sum_{i=k}^{\ell} \check{s}_{i}\right\} .
$$

Then the output of $D(D(\mathbf{b}, R(\mathbf{a}, \mathbf{s})), D(\mathbf{a}, \mathbf{s}))$ is the increment sequence of

$$
\widetilde{H}_{m}=\sup _{\ell \leq m}\left\{\check{B}_{\ell}+\sum_{j=\ell}^{m} \widetilde{a}_{j}\right\}=\sup _{k \leq m}\left\{B_{k}+\max _{\ell: k \leq \ell \leq m}\left[\sum_{i=k}^{\ell} \check{s}_{i}+\sum_{j=\ell}^{m} \widetilde{a}_{j}\right]\right\} .
$$

It remains to check that

$$
\max _{\ell: k \leq \ell \leq m}\left[\sum_{i=k}^{\ell} \check{s}_{i}+\sum_{j=\ell}^{m} \widetilde{a}_{j}\right]=\max _{\ell: k \leq \ell \leq m}\left[\sum_{i=k}^{\ell} a_{i}+\sum_{j=\ell}^{m} s_{j}\right] .
$$

This can be verified with a case-by-case analysis. See Lemma 4.3 in [15].

Specialize to stationary $\mathrm{M} / \mathrm{M} / 1$ queues. Let $\sigma$ be a service rate and $\alpha_{1}, \alpha_{2}$ arrival rates. Assume $\sigma>\alpha_{1}>\alpha_{2}>0$. Let $\mathbf{b}^{1}, \mathbf{b}^{2}, \mathbf{s}$ be mutually independent i.i.d. sequences with marginals $b_{j}^{k} \sim \operatorname{Exp}\left(\alpha_{k}\right)$ for $k \in\{1,2\}$ and $s_{j} \sim \operatorname{Exp}(\sigma)$. Define a jointly distributed pair of arrival sequences by $\left(\mathbf{a}^{1}, \mathbf{a}^{2}\right)=$ $\left(\mathbf{b}^{1}, D\left(\mathbf{b}^{2}, \mathbf{b}^{1}\right)\right)$. From these and services $\mathbf{s}$, define jointly distributed output variables:

$$
\mathbf{d}^{k}=D\left(\mathbf{a}^{k}, \mathbf{s}\right), \quad \mathbf{t}^{k}=S\left(\mathbf{a}^{k}, \mathbf{s}\right), \quad \text { and } \quad \check{\mathbf{s}}^{k}=R\left(\mathbf{a}^{k}, \mathbf{s}\right) \quad \text { for } k \in\{1,2\} .
$$

Lemma A.2. We have the following properties.

(i) Marginally $\mathbf{a}^{2}$ is a sequence of i.i.d. $\operatorname{Exp}\left(\alpha_{2}\right)$ variables.

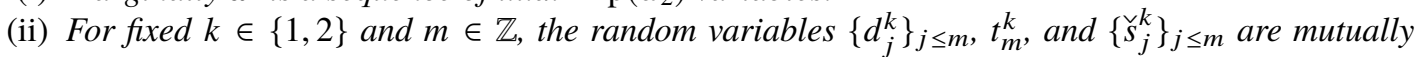
independent with marginal distributions $d_{j}^{k} \sim \operatorname{Exp}\left(\alpha_{k}\right), t_{m}^{k} \sim \operatorname{Exp}\left(\sigma-\alpha_{k}\right)$, and $\varsigma_{j}^{k} \sim \operatorname{Exp}(\sigma)$.

(iii) For a fixed $k \in\{1,2\}$, sequences $\mathbf{d}^{k}$ and $\breve{\mathbf{s}}^{k}$ are mutually independent sequences of i.i.d. random variables with marginal distributions $d_{j}^{k} \sim \operatorname{Exp}\left(\alpha_{k}\right)$ and $\breve{s}_{j}^{k} \sim \operatorname{Exp}(\sigma)$.

(iv) $\left(\mathbf{d}^{1}, \mathbf{d}^{2}\right) \stackrel{d}{=}\left(\mathbf{a}^{1}, \mathbf{a}^{2}\right)$ : in other words, we have a distributional fixed point for this joint queueing operator.

(v) For any $m \in \mathbb{Z}$, the random variables $\left\{a_{i}^{2}\right\}_{i \leq m}$ and $\left\{a_{j}^{1}\right\}_{j \geq m+1}$ are mutually independent.

Proof. Parts (i)-(iii) are basic M/M/1 queueing theory. Proofs can be found, for example, in Lemma B.2 in Appendix B of [15].

For part (iv), the marginal distributions of $\mathbf{d}^{1}$ and $\mathbf{d}^{2}$ are the correct ones by Lemma A.2(iii). To establish the correct joint distribution, the definition of $\left(\mathbf{a}^{1}, \mathbf{a}^{2}\right)$ points us to find an i.i.d. $\operatorname{Exp}\left(\alpha_{2}\right)$ random sequence $\mathbf{z}$ that is independent of $\mathbf{d}^{1}$ and satisfies $\mathbf{d}^{2}=D\left(\mathbf{z}, \mathbf{d}^{1}\right)$. From the definitions and Lemma A.1,

$$
\mathbf{d}^{2}=D\left(\mathbf{a}^{2}, \mathbf{s}\right)=D\left(D\left(\mathbf{b}^{2}, \mathbf{a}^{1}\right), \mathbf{s}\right)=D\left(D\left(\mathbf{b}^{2}, R\left(\mathbf{a}^{1}, \mathbf{s}\right)\right), D\left(\mathbf{a}^{1}, \mathbf{s}\right)\right)=D\left(D\left(\mathbf{b}^{2}, \check{\mathbf{s}}^{1}\right), \mathbf{d}^{1}\right) .
$$

By assumption, $\mathbf{b}^{2}, \mathbf{a}^{1}, \mathbf{s}$ are independent. Hence, by Lemma A.2(iii), $\mathbf{b}^{2}, \check{\mathbf{s}}^{1}, \mathbf{d}^{1}$ are independent. So we take $\mathbf{z}=D\left(\mathbf{b}^{2}, \breve{\mathbf{s}}^{1}\right)$, which is an i.i.d. $\operatorname{Exp}\left(\alpha_{2}\right)$ sequence by Lemma A.2(iii). This proves part (iv).

We know that marginally $\mathbf{a}^{1}$ and $\mathbf{a}^{2}$ are i.i.d. sequences. In queueing language, observation (v) becomes obvious. Namely, since $\mathbf{a}^{2}=D\left(\mathbf{b}^{2}, \mathbf{a}^{1}\right)$, the statement is that past inter-departure times $\left\{a_{i}^{2}\right\}_{i \leq m}$ are independent of future inter-arrival times $\left\{a_{j}^{1}\right\}_{j \geq m+1}$. Rigorously, (A.2) and (A.3) show that variables $\left\{a_{i}^{2}\right\}_{i \leq m}$ are functions of $\left(\left\{b_{i}^{2}\right\}_{i \leq m},\left\{a_{i}^{1}\right\}_{i \leq m}\right)$, which are independent of $\left\{a_{j}^{1}\right\}_{j \geq m+1}$. 


\section{Appendix B. Coupling and monotonicity in last-passage percolation}

In this section, $\omega=\left(\omega_{x}\right)_{x \in \mathbb{Z}^{2}}$ is a fixed assignment of real weights. $G_{x, y}$ is the last-passage value defined by (1.1). No probability is involved.

Lemma B.1. Suppose weights $\omega$ and $\widetilde{\omega}$ satisfy $\omega_{o+i e_{1}} \geq \widetilde{\omega}_{o+i e_{1}}, \omega_{o+j e_{2}} \leq \widetilde{\omega}_{o+j e_{2}}$, and $\omega_{x}=\widetilde{\omega}_{x}$ for $i, j \geq 1$ and $x \in o+\mathbb{Z}_{>0}^{2}$. As in (1.1), define LPP processes

$$
G_{o, y}=\max _{x_{\bullet} \in \Pi_{o, y}} \sum_{k=0}^{|y-o|_{1}} \omega_{x_{k}} \text { and } \widetilde{G}_{o, y}=\max _{x_{\bullet} \in \Pi_{o, y}} \sum_{k=0}^{|y-o|_{1}} \widetilde{\omega}_{x_{k}} \text { for } y \in o+\mathbb{Z}_{\geq 0}^{2} .
$$

Then for all $y \in o+\mathbb{Z}_{\geq 0}^{2}$, the increments over nearest-neighbor edges satisfy

$$
G_{o, y+e_{1}}-G_{o, y} \geq \widetilde{G}_{o, y+e_{1}}-\widetilde{G}_{o, y} \text { and } G_{o, y+e_{2}}-G_{o, y} \leq \widetilde{G}_{o, y+e_{2}}-\widetilde{G}_{o, y} .
$$

Proof. The statements are true by construction for edges $\left(y, y+e_{i}\right)$ that lie on the axes $o+\mathbb{Z}_{\geq 0} e_{i}$. Proceed by induction: assuming the inequalities hold for the edges $\left(y, y+e_{2}\right)$ and $\left(y, y+e_{1}\right)$, deduce them for the edges $\left(y+e_{2}, y+e_{1}+e_{2}\right)$ and $\left(y+e_{1}, y+e_{1}+e_{2}\right)$.

Lemma B.2 (Crossing Lemma). The inequalities below are valid whenever the last-passage values are defined.

$$
\begin{aligned}
& G_{o+e_{1}, x+e_{2}}-G_{o+e_{1}, x} \leq G_{o, x+e_{2}}-G_{o, x} \leq G_{o+e_{2}, x+e_{2}}-G_{o+e_{2}, x} \\
& G_{o+e_{2}, x+e_{1}}-G_{o+e_{2}, x} \leq G_{o, x+e_{1}}-G_{o, x} \leq G_{o+e_{1}, x+e_{1}}-G_{o+e_{1}, x} .
\end{aligned}
$$

Proof. The proofs of all parts are similar. We prove the second inequality in (B.1): that is,

$$
G_{o, x+e_{2}}-G_{o, x} \leq G_{o+e_{2}, x+e_{2}}-G_{o+e_{2}, x} .
$$

The geodesics $\pi_{o, x+e_{2}}$ and $\pi_{o+e_{2}, x}$ must cross. Let $u$ be the first point where they meet. Note that

$$
G_{o, u}+G_{u, x} \leq G_{o, x} \quad \text { and } \quad G_{o+e_{2}, u}+G_{u, x+e_{2}} \leq G_{o+e_{2}, x+e_{2}} .
$$

Add the two inequalities in (B.3), and rearrange to obtain (B.2).

This inequality can also be proved from Lemma B.1, by writing $G_{o+e_{2}, x+e_{2}}-G_{o+e_{2}, x}=\widetilde{G}_{o, x+e_{2}}-$ $\widetilde{G}_{o, x}$ with environment $\widetilde{\omega}_{o+y}=\omega_{o+y}$ when $y_{2}>0$ and $\widetilde{\omega}_{o+i e_{1}}=-M$ for large enough $M$.

Fix base points $u \leq v$ on $\mathbb{Z}^{2}$. On the quadrant $v+\mathbb{Z}_{\geq 0}^{2}$, put a corner weight $\eta_{v}=0$ and define boundary weights

$$
\eta_{v+k e_{i}}=G_{u, v+k e_{i}}-G_{u, v+(k-1) e_{i}} \text { for } k \in \mathbb{Z}_{>0} \text { and } i \in\{1,2\} .
$$

In the bulk, use $\eta_{x}=\omega_{x}$ for $x \in v+\mathbb{Z}_{>0}^{2}$. Denote the LPP process in $v+\mathbb{Z}_{\geq 0}^{2}$ that uses weights $\left\{\eta_{x}\right\}_{x \in v+\mathbb{Z}_{\geq 0}^{2}}$ by

$$
G_{v, x}^{[u]}=\max _{x_{\bullet} \in \Pi_{v, x}} \sum_{i=0}^{|x-v|_{1}} \eta_{x_{i}}, \quad x \in v+\mathbb{Z}_{\geq 0}^{2} .
$$

The superscript $[u]$ indicates that $G^{[u]}$ uses boundary weights determined by the process $G_{u}$, with base point $u$. Figure B.1 illustrates the next lemma. The proof of the lemma is elementary.

Lemma B.3. Let $u \leq v \leq y$ in $\mathbb{Z}^{2}$. Then $G_{u, y}=G_{u, v}+G_{v, y}^{[u]}$. The restriction of any geodesic of $G_{u, y}$ to $v+\mathbb{Z}_{\geq 0}^{2}$ is part of a geodesic of $G_{v, y}^{[u]}$. The edges with one endpoint in $v+\mathbb{Z}_{>0}^{2}$ that belong to a geodesic of $G_{v, y}^{[u]}$ extend to a geodesic of $G_{u, y}$. 


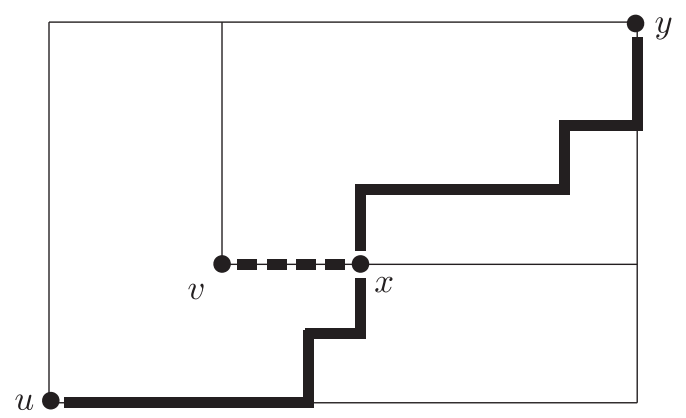

Figure B.1. Illustration of Lemma B.3. Path $u-x-y$ is a geodesic of $G_{u, y}$, and path $v-x-y$ is a geodesic of $G_{v, y}^{[u]}$.

Assume now that the weights are such that geodesics are unique. Define the exit point $Z_{u, p}$ as in (4.1). For $k \geq 1$, let $Z_{u+k e_{1}, p}^{[u]}$ be the exit point of the geodesic of $G_{u+k e_{1}, p}^{[u]}$. The lemma below follows from taking $v=u+k e_{1}$ in Lemma B.3.

Lemma B.4. For positive integers $m, Z_{u, p}=k+m$ if and only if $Z_{u+k e_{1}, p}^{[u]}=m$.

\section{Appendix C. Random walk bounds}

Lemma C.1. Let $\alpha>\beta>0$, and let $S_{n}=\sum_{k=1}^{n} Z_{k}$ be a random walk with step distribution $Z_{k} \sim$ $\operatorname{Exp}(\alpha)-\operatorname{Exp}(\beta)$ (difference of two independent exponentials). Then there is an absolute constant $C$ independent of all the parameters such that for $n \in \mathbb{Z}_{>0}$,

$$
\mathbb{P}\left(S_{1}>0, S_{2}>0, \ldots, S_{n}>0\right) \leq \frac{C}{\sqrt{n}}\left(1-\frac{(\alpha-\beta)^{2}}{(\alpha+\beta)^{2}}\right)^{n}
$$

and

$$
\mathbb{P}\left(S_{1}<0, S_{2}<0, \ldots, S_{n}<0\right) \leq \frac{C}{\sqrt{n}}\left(1-\frac{(\alpha-\beta)^{2}}{(\alpha+\beta)^{2}}\right)^{n}+\frac{\alpha-\beta}{\alpha} .
$$

Proof. Define the events

$$
A_{n}^{\alpha, \beta}=\left\{S_{1}>0, \ldots, S_{n}>0\right\} \quad \text { and } \quad B_{n}^{\alpha, \beta}=\left\{S_{1}>0, \ldots, S_{n-1}>0, S_{n}<0\right\}
$$

for $n \in \mathbb{Z}_{>0}$ and also the decreasing limit $A_{\infty}^{\alpha, \beta}=\bigcap_{n \geq 1} A_{n}^{\alpha, \beta}$. Then

$$
P\left(A_{n}^{\alpha, \beta}\right)=\sum_{k=n+1}^{\infty} P\left(B_{k}^{\alpha, \beta}\right)+P\left(A_{\infty}^{\alpha, \beta}\right) .
$$

Lemma B.3 in Appendix B of [15] calculated

$$
P\left(B_{n}^{\alpha, \beta}\right)=C_{n-1} \frac{\alpha^{n} \beta^{n-1}}{(\alpha+\beta)^{2 n-1}}
$$

where $C_{n}=\frac{1}{n+1}\left(\begin{array}{c}2 n \\ n\end{array}\right), n \geq 0$, are the Catalan numbers. Note that parameters $\alpha$ and $\beta$ are switched around here compared with Lemma B.3 of [15]. From $\left(\begin{array}{c}2 n \\ n\end{array}\right) 2^{-2 n} \sim(\pi n)^{-1 / 2}$, we can fix a constant $c_{0}$ such that $C_{k-1} \leq c_{0} 4^{k-1} k^{-3 / 2}$. 
The assumption $\alpha>\beta$ gives $E Z_{k}=\alpha^{-1}-\beta^{-1}<0$, and hence $\sum_{n \geq 1} P\left(B_{n}^{\alpha, \beta}\right)=1$ and $P\left(A_{\infty}^{\alpha, \beta}\right)=0$. Thus (C.2) and (C.3), together with $\sum_{k=n+1}^{\infty} k^{-3 / 2} \leq 2 n^{-1 / 2}$, give

$$
\begin{aligned}
P\left(A_{n}^{\alpha, \beta}\right) & =\frac{\alpha}{\alpha+\beta} \sum_{k=n+1}^{\infty} C_{k-1}\left(\frac{\alpha \beta}{(\beta+\alpha)^{2}}\right)^{k-1} \leq \frac{c_{0} \alpha}{\alpha+\beta} \sum_{k=n+1}^{\infty} k^{-3 / 2}\left(1-\frac{(\alpha-\beta)^{2}}{(\alpha+\beta)^{2}}\right)^{k-1} \\
& \leq \frac{2 c_{0} \alpha}{\alpha+\beta} \cdot \frac{1}{\sqrt{n}}\left(1-\frac{(\alpha-\beta)^{2}}{(\alpha+\beta)^{2}}\right)^{n} .
\end{aligned}
$$

Since $-S_{n}$ is obtained from $S_{n}$ by switching $\alpha$ and $\beta$ around,

$$
P\left(S_{1}<0, \ldots, S_{n}<0\right)=P\left(A_{n}^{\beta, \alpha}\right)=\sum_{k=n+1}^{\infty} P\left(B_{k}^{\beta, \alpha}\right)+P\left(A_{\infty}^{\beta, \alpha}\right) .
$$

Bound the series above as in (C.4) (with $\alpha$ and $\beta$ interchanged), and add $P\left(A_{\infty}^{\beta, \alpha}\right)=\frac{\alpha-\beta}{\alpha}$. This last fact appears on page 600 of Resnick [32] and in Example VI.8(b) on page 193 of Feller II [16].

Acknowledgments. M. Balázs was partially supported by EPSRC's EP/R021449/1 Standard Grant.

O. Busani was supported by EPSRC's EP/R021449/1 Standard Grant.

T. Seppäläinen was partially supported by National Science Foundation grant DMS-1854619 and by the Wisconsin Alumni Research Foundation.

The authors would like to acknowledge EPSRC grant number EP/R021449/1.

The authors thank F. Rassoul-Agha and S. Groathouse for useful comments.

Conflict of Interest: None.

\section{References}

[1] Daniel Ahlberg and Christopher Hoffman, 'Random coalescing geodesics in first-passage percolation', arXiv:1609.02447, 2016.

[2] David Aldous and Persi Diaconis, 'Hammersley's interacting particle process and longest increasing subsequences', Probab. Theory Related Fields, 103(2): 199-213, 1995.

[3] Antonio Auffinger, Michael Damron, and Jack Hanson, 'Limiting geodesics for first-passage percolation on subsets of $\mathbb{Z}^{2}$, Ann. Appl. Probab., 25(1): 373-405, 2015.

[4] Antonio Auffinger, Michael Damron, and Jack Hanson, 50 years of first-passage percolation, volume $\mathbf{6 8}$ of University Lecture Series, (American Mathematical Society, Providence, RI, 2017).

[5] Márton Balázs, Eric Cator, and Timo Seppäläinen, 'Cube root fluctuations for the corner growth model associated to the exclusion process', Electron. J. Probab., 11(42): 1094-1132 (electronic), 2006.

[6] Márton Balázs, Júlia Komjáthy, and Timo Seppäläinen, 'Microscopic concavity and fluctuation bounds in a class of deposition processes', Ann. Inst. Henri Poincaré Probab. Stat., 48(1): 151-187, 2012.

[7] Riddhipratim Basu, Christopher Hoffman, and Allan Sly, 'Nonexistence of bigeodesics in integrable models of last passage percolation', arXiv: 1811.04908, 2018.

[8] Riddhipratim Basu, Vladas Sidoravicius, and Allan Sly, 'Last passage percolation with a defect line and the solution of the slow bond problem', arXiv:1408.3464, 2014.

[9] Eric Cator and Piet Groeneboom, 'Second class particles and cube root asymptotics for Hammersley's process', Ann. Probab., 34(4): 1273-1295, 2006.

[10] Hans Chaumont and Christian Noack, 'Characterizing stationary $1+1$ dimensional lattice polymer models', Electron. J. Probab., 23:Paper 38, 19, 2018.

[11] Michael Damron and Jack Hanson, 'Busemann functions and infinite geodesics in two-dimensional first-passage percolation', Comm. Math. Phys., 325(3): 917-963, 2014.

[12] Michael Damron and Jack Hanson, 'Bigeodesics in first-passage percolation', Comm. Math. Phys., 349(2): 753-776, 2017.

[13] Duncan Dauvergne, Janosch Ortmann, and Bálint Virág, 'The directed landscape', arXiv:1812.00309, 2018.

[14] Murray Eden, 'A two-dimensional growth process', In Proc. 4th Berkeley Sympos. Math. Statist. and Prob., Vol. IV (Univ. California Press, Berkeley, Calif., 1961), 223-239.

[15] Wai-Tong (Louis) Fan and Timo Seppäläinen, 'Joint distribution of Busemann functions in the exactly solvable corner growth model', Journal Probability and Mathematical Physics, arXiv:1808.09069, 2018. 
[16] William Feller, An introduction to probability theory and its applications, Vol. II., 2e (John Wiley \& Sons Inc., New York, 1971).

[17] Nicos Georgiou, Firas Rassoul-Agha, and Timo Seppäläinen, 'Geodesics and the competition interface for the corner growth model', Probab. Theory Related Fields, 169(1-2): 223-255, 2017.

[18] Nicos Georgiou, Firas Rassoul-Agha, and Timo Seppäläinen, 'Stationary cocycles and Busemann functions for the corner growth model', Probab. Theory Related Fields, 169(1-2): 177-222, 2017.

[19] Janko Gravner, Craig A. Tracy, and Harold Widom, 'Limit theorems for height fluctuations in a class of discrete space and time growth models', J. Statist. Phys., 102(5-6): 1085-1132, 2001.

[20] John M. Hammersley and Dominic J. A. Welsh, 'First-passage percolation, subadditive processes, stochastic networks, and generalized renewal theory', In Proc. Internat. Res. Semin., Statist. Lab., Univ. California, Berkeley, Calif, (Springer-Verlag, New York, 1965), 61-110.

[21] Christopher Janjigian and Firas Rassoul-Agha, 'Busemann functions and Gibbs measures in directed polymer models on $\mathbb{Z}^{2}$, Ann. Probab., 48(2): 778-816, 2020.

[22] Christopher Janjigian, Firas Rassoul-Agha, and Timo Seppäläinen, 'Geometry of geodesics through Busemann measures in directed last-passage percolation', arXiv:1908.09040, 2019.

[23] Kurt Johansson, 'Shape fluctuations and random matrices', Comm. Math. Phys., 209(2): 437-476, 2000.

[24] Kurt Johansson, 'Discrete orthogonal polynomial ensembles and the Plancherel measure', Ann. of Math. (2), 153(1): 259296, 2001.

[25] Harry Kesten, 'Aspects of first passage percolation', In École d'été de probabilités de Saint-Flour, XIV_-1984, volume 1180 of Lecture Notes in Math. (Springer, Berlin, 1986), 125-264.

[26] Cristina Licea and Charles M. Newman, 'Geodesics in two-dimensional first-passage percolation', Ann. Probab., 24(1): 399-410, 1996.

[27] Konstantin Matetski, Jeremy Quastel, and Daniel Remenik, 'The KPZ fixed point', arXiv preprint arXiv:1701.00018, 2016.

[28] Charles M. Newman, 'A surface view of first-passage percolation', In Proceedings of the International Congress of Mathematicians, Vol. 1, 2 (Zürich, 1994) (Birkhäuser, Basel, 1995), 1017-1023.

[29] Charles M. Newman, Topics in Disordered Systems, Lectures in Mathematics ETH Zürich, (Birkhäuser Verlag, Basel, 1997).

[30] Neil O'Connell and Marc Yor, 'Brownian analogues of Burke's theorem', Stochastic Process. Appl., 96(2): 285-304, 2001.

[31] Leandro P. R. Pimentel, 'Duality between coalescence times and exit points in last-passage percolation models', Ann. Probab., 44(5): 3187-3206, 2016.

[32] Sidney Resnick, Adventures in Stochastic Processes, (Birkhäuser Boston Inc., Boston, MA, 1992).

[33] Timo Seppäläinen, 'Increasing sequences of independent points on the planar lattice', Ann. Appl. Probab., 7(4): 886-898, 1997.

[34] Timo Seppäläinen, 'Exact limiting shape for a simplified model of first-passage percolation on the plane', Ann. Probab., 26(3): 1232-1250, 1998.

[35] Timo Seppäläinen, 'The corner growth model with exponential weights', In Random Growth Models, volume 75 of Proc. Sympos. Appl. Math. (Amer. Math. Soc., Providence, RI, 2018), 133-201, arXiv:1709.05771.

[36] Jan Wehr and Jung Woo, 'Absence of geodesics in first-passage percolation on a half-plane', Ann. Probab., 26(1): 358-367, 1998. 